\title{
DISTRIBUIÇÃO DE METAIS NAS ÁGUAS DO RIO CABEÇA, TRIBUTÁRIO NA BACIA DO RIO CORUMBATAÍ, SP
}

\section{DISTRIBUTION OF METALS IN CABEÇA RIVER WATERS, TRIBUTARY AT THE CORUMBATAÍ RIVER BASIN, SP}

\section{Eduardo Beraldo de Morais ${ }^{1}$, Sâmia Maria Tauk-Tornisielo ${ }^{2}$, Alexandre Augusto Oliveira Santos ${ }^{2}$, Silvia Elena Ventorini ${ }^{3}$}
${ }^{1}$ Professor Adjunto, Departamento de Engenharia Sanitária e Ambiental, DESA, Universidade Federal de Mato Grosso, UFMT, Campus de Cuiabá, Avenida Fernando Correa da Costa, 2367, CEP 78060-900, Cuiabá, MT. E-mail: ebmorais@ufmt.br
${ }^{2}$ Pesquisadora do Centro de Estudos Ambientais, CEA, Universidade Estadual Paulista, UNESP, Campus de Rio Claro, Avenida 24-A, 1515, Bela Vista, CEP 13506-900, Rio Claro, SP. E-mail: seb@rc.unesp.br / alexbio2002@yahoo.com.br ${ }^{3}$ Professora Assistente, Departamento de Geografia, Universidade Federal São João Del Rei, Av. Visconde do Rio Preto, s/n Colônia do Bengo, CEP 36300-000, São João Del Rei, MG. E-mail: sventorini@ufsj.edu.br

\begin{abstract}
RESUMO
Amostras de água foram coletadas, com frequência mensal no período de abril de 2007 a março de 2009, em cinco pontos de amostragem no rio Cabeça e dois pontos no rio Passa Cinco, ambos tributários na bacia do rio Corumbataí, $\mathrm{SP}$, para avaliar a distribuição dos metais $\mathrm{Al}, \mathrm{Cd}, \mathrm{Cr}, \mathrm{Cu}, \mathrm{Fe}$, $\mathrm{Mn}, \mathrm{Ni}, \mathrm{Pb}, \mathrm{Zn}, \mathrm{Ca}$ e $\mathrm{Mg}$. Esses elementos foram quantificados por meio de espectrometria de emissão atômica e os resultados foram analisados pela técnica estatística multivariada (análise de componentes principais), relacionando os valores com os períodos seco e chuvoso. Os metais $\mathrm{Cr}$, $\mathrm{Cu}$ e $\mathrm{Ni}$ não foram detectados e o $\mathrm{Cd}$ e o $\mathrm{Pb}$ foram detectados em 8,3\% e 10,1\% das amostras, respectivamente, e os valores estavam acima do permitido pela Resolução CONAMA 357/2005 para rios de classe 2. Também estavam em desacordo com essa legislação os valores de Fe e 36,9\% dos valores encontrados para o Al. Esses dois metais são fortemente influenciados pelas características pedológicas da área estudada. As concentrações de $\mathrm{Zn}$ não extrapolaram os limites, mas a presença desse elemento pode estar relacionada com a falta de matas ciliares e intensa ocupação agrícola, principalmente com a cultura canavieira. O Mn teve seus valores abaixo do limite estabelecido pela legislação vigente, com exceção de seis amostras. Os maiores valores de $\mathrm{Ca}$ foram observados no período chuvoso, nos pontos de amostragens situados em áreas de exploração de calcário dolomítico e cultivo de cana-de-açúcar na qual a calagem é frequente.

Palavras chave: Microbacia. Atividades Agropecuárias. Análise de Componentes Principais. Canade-açúcar.
\end{abstract}




\begin{abstract}
Water samples were collected with monthly frequency from April 2007 to March 2009 at five sampling points in the Cabeça River and two points in Passa Cinco River, both tributaries at the Corumbataí River Basin, SP, and the distribution of metals $\mathrm{Al}, \mathrm{Cd}, \mathrm{Cr}, \mathrm{Cu}, \mathrm{Fe}, \mathrm{Mn}, \mathrm{Ni}, \mathrm{Pb}, \mathrm{Zn}, \mathrm{Ca}$ and $\mathrm{Mg}$ was evaluate. These elements were quantified by atomic emission spectrometry and the results were analyzed by multivariate statistical technique (principal component analysis), relating the values with the dry and rainy seasons. The metals $\mathrm{Cr}, \mathrm{Cu}$ and $\mathrm{Ni}$ were not detected and $\mathrm{Cd}$ and $\mathrm{Pb}$ were detected in $8.3 \%$ and $10.1 \%$ of the samples, respectively, and the values were above those permitted by CONAMA Resolution 357/2005 for Class 2 Rivers. The values of Fe and 36.9\% of the values for the $\mathrm{Al}$ also exceeded the limit established by legislation. These two metals are strongly influenced by soil characteristics of the studied area. Zn concentrations were below the limit from standard from CONAMA Resolution, but the presence of this element can be related to the lack of riparian vegetation and intense agricultural occupation, especially with sugar cane. Only six samples have values above the limit established by legislation. The highest values of $\mathrm{Ca}$ were observed in the rainy season in sampling points located in areas of exploitation of lime and sugar cane cultivation in which liming is frequent.
\end{abstract}

Keywords: Watershed. Agricultural Activities. Principal Component Analysis. Sugar cane.

\title{
1. INTRODUÇÃO
}

A poluição de solos e das águas por metais pesados e pesticidas e a eutrofização decorrente do uso intensivo de fertilizantes e despejos de poluentes orgânicos são alguns problemas detectados em bacias hidrográficas de uso rural (RAMALHO et al., 2000; CORBI et al., 2006; RAMOS et al., 2006; RIBBE et al., 2008). Estes poluentes atingem os corpos de águas por meio de fontes pontuais ou devido ao escoamento superficial na área de drenagem, sendo este último fator atuante principalmente quando as matas ciliares não estão conservadas. Para a eficiente gestão e conservação dessas unidades é necessária a realização do diagnóstico ambiental nestas áreas (HOSONO et al., 2007).

A poluição de ambientes aquáticos por metais pesados em especial, causa grandes preocupações devido os efeitos tóxicos ao homem e demais seres vivos, abundância, persistência e acúmulo de tais elementos no ambiente (ZHOU et al., 2008; VAROL e SEN, 2012). Dessa forma, diversos pesquisadores têm dispensado esforços na quantificação e comportamento de metais nos ecossistemas aquáticos (RYBICKA et al., 2005; PERTSEMLI e VOUTSA, 2007; KAUSHIK et al., 2009; LI e ZHANG, 2010a; MECHE et al., 2010; MARTIN, 2012).

Os metais podem ser introduzidos nos corpos hídricos como resultados de processos naturais ou antropogênicos. Por um lado, fontes naturais envolvem fenômenos como intemperismo, erosão, erupções vulcânicas e deposição atmosférica (LI et al., 2008; LI e ZHANG, 2010b) e normalmente em ambientes poucos afetados pela atividade humana, as concentrações da maioria dos metais nos corpos hídricos superficiais são baixas e originadas de rochas e solos (REZA e SINGH, 2010). Por outro lado, as principais fontes antropogênicas da contaminação de ambientes aquáticos por estes elementos são efluentes domésticos (MACEDA-VEIGA et al., 2012), efluentes industriais (KRISHNA et al., 2009, SUTHAR et al., 2009), atividades mineradoras (HE et al., 1997; KIM et al., 2007) e atividades agrícolas (TANG et al., 2010, LU et al., 2012).

Os insumos agrícolas como pesticidas, fertilizantes comerciais e demais corretivos são alguns produtos utilizados na agricultura que podem ocasionar poluição por metais. $\mathrm{O}$ uso intensivo de agrotóxicos, por exemplo, pode introduzir metais no ambiente, pois estes fazem parte do princípio ativo dos metalo-pesticidas (GIMENO-GARCÍA et al., 1996). Os fertilizantes, por sua vez, derivados principalmente de rochas fosfáticas apresentam tais elementos como impurezas em 
níveis traço dependendo de sua origem geológica e localização (AL-SHAWI e DAHL, 1999; AYDIN et al., 2010).

Estudos têm demonstrado o acúmulo de metais no solo e plantas como $\mathrm{Cu}, \mathrm{Cd}, \mathrm{Ni}, \mathrm{Zn}, \mathrm{Co}$, $\mathrm{Pb}, \mathrm{Mn}, \mathrm{Fe}$, As e $\mathrm{Cr}$ em decorrência do uso intensivo de fertilizantes (AL-SHAWI e DAHL, 1999; NZIGUHEBA e SMOLDE, 2008; SABIHA-JAVIED et al., 2009; AYDIN et al., 2010; ZHANG e ZHANG, 2010; EL-TAHER e ALTHOYAIB, 2012). O impacto, entretanto, do acúmulo de metais pesados decorrente da agricultura intensiva na bacia hidrográfica como um todo, incluindo a deterioração da qualidade da água, ainda é pouco compreendido (ZHANG e SHAN, 2008, TANG et al., 2010).

A bacia hidrográfica do rio Corumbataí, SP, tem destaque social e econômico devido à capacidade de abastecimento de diversas cidades como Analândia, Corumbataí, Rio Claro, Santa Gertrudes, Ipeúna, entre outras. Permite ainda a exportação de água para o município de Piracicaba localizado na bacia vizinha, a qual apresenta sérios problemas com poluição de seus corpos hídricos. Destacam-se na bacia do rio Corumbataí os afluentes Ribeirão Claro e Passa Cinco, além do rio Cabeça, afluente deste último. Nesta bacia é verificada grande influência antrópica com destaques para atividades industriais e agropecuárias. O presente trabalho teve por objetivo avaliar a distribuição espaço-temporal de metais nas águas do rio Cabeça, cuja microbacia é utilizada para o desenvolvimento de atividades agropecuárias, com destaque para a cultura de cana de açúcar e pastagens. Verificou-se ainda a contribuição do rio Cabeça no sentido de alterar a concentração dos metais estudados no rio Passa Cinco, corpo hídrico receptor de suas águas. Este estudo também contribuirá com informações para a melhor compreensão da contribuição das atividades agrícolas na poluição dos corpos hídricos superficiais por metais. E contribuir com informações para a melhor compreensão da contribuição das atividades agrícolas na poluição dos corpos hídricos superficiais por metais.

\section{MATERIAL E MÉTODOS}

\subsection{Descrição da área de estudo e pontos de amostragem}

A área de estudo, microbacia do rio Cabeça possui aproximadamente $214 \mathrm{~km}^{2}$ de extensão, sendo $30,5 \%$ de sua área utilizada para a cultura de cana de açúcar e 39,1\% para pastagem (MORAIS, 2010). Ao longo deste rio foram estabelecidos cinco pontos de amostragens (P1 a P5) definidos de acordo com a facilidade de acesso e caracterização da microbacia hidrográfica quanto aos seus aspectos geomorfológicos, geológicos e principalmente atividades agrícolas desenvolvidas. Além destes cinco pontos e com o intuito de verificar a influência das águas do rio Cabeça nos parâmetros avaliados, ou seja, metais nas águas do rio Passa Cinco, outros dois pontos foram estabelecidos neste último rio sendo o P6 anterior ao deságue do rio Cabeça e o P7 localizado aproximadamente três quilômetros após o Passa Cinco receber as águas desse rio (Figura 1 e Tabela 1). 


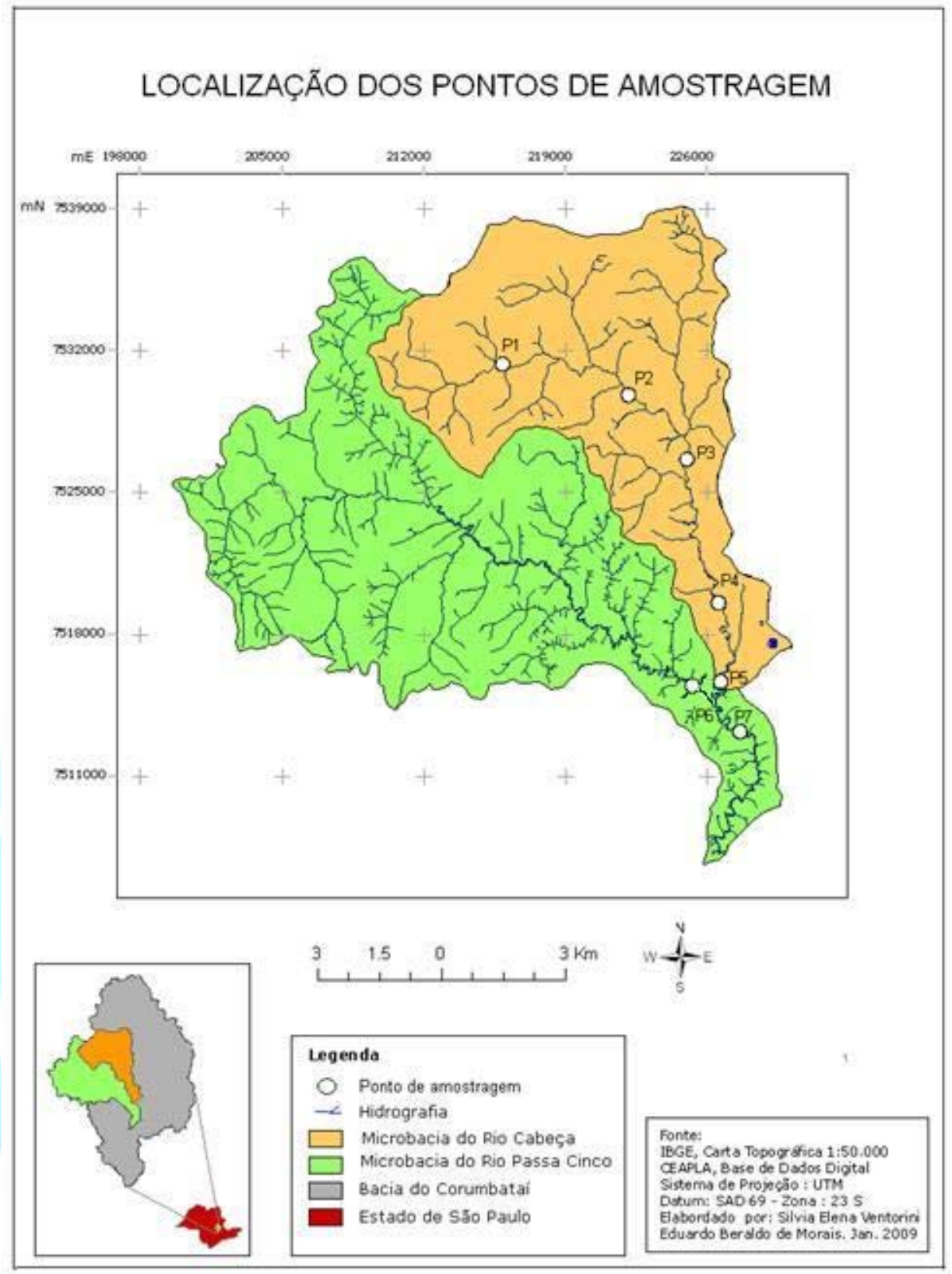

Figura 1. Localização da área de estudo na bacia do rio Corumbataí e pontos de amostragens.

Tabela 1 - Pontos de amostragens e suas características na área de estudo.

\begin{tabular}{lccc}
\hline Ponto & Corpo hídrico & Localização & Uso do solo \\
\hline P1 & Rio Cabeça & $215866 \mathrm{mE}-7531682 \mathrm{mN}$ & Pastagem \\
P2 & Rio Cabeça & $222080 \mathrm{mE}-7529818 \mathrm{mN}$ & Pastagem \\
P3 & Rio Cabeça & $224960 \mathrm{mE}-7526640 \mathrm{mN}$ & Pastagem e Cana-de-açúcar \\
P4 & Rio Cabeça & $226518 \mathrm{mE}-7526640 \mathrm{mN}$ & Cana-de-açúcar \\
P5 & Rio Cabeça & $226518 \mathrm{mE}-7519564 \mathrm{mN}$ & Cana-de-açúcar \\
P6 & Rio Passa-Cinco & $226388 \mathrm{mE}-7519564 \mathrm{mN}$ & Cana-de-açúcar \\
P7 & Rio Passa-Cinco & $227576 \mathrm{mE}-7513221 \mathrm{mN}$ & Cana-de-açúcar \\
\hline
\end{tabular}

Fonte: Morais (2010). 
Os pontos amostrais P1 e P2 destacaram-se por apresentar o predomínio de atividades de pastagem em suas áreas adjacentes, atividades estas que foram uma das responsáveis pela supressão da mata ciliar nestes pontos, pois foram constatados que o pisoteamento do gado durante a dessedentação impedia a recuperação da vegetação ripária. Merece destacar ainda que nestas localidades foi observado intenso assoreamento principalmente no ponto P2 (Figura 2) cuja profundidade do corpo hídrico não ultrapassou $50 \mathrm{~cm}$ e foi contatado grande quantidade de areia em seu leito. É possível observar também na Figura 2 a ausência da vegetação ripária e instabilidade das margens, situação que se torna mais crítica no período de maior pluviosidade.

O ponto amostral P3 foi escolhido por estar localizado em uma área de transição entre a atividade de pastagem e plantações de cana de açúcar. Ainda neste ponto verificou-se um pequeno represamento no curso do rio para facilitar a passagem de veículos automotores uma vez a ponte existente no local estava desativada. Esse represamento foi feito por meio de manilhas de concreto que permitiam o escoamento das águas e entulho de construção civil. A diminuição da vazão neste ponto decorrente de represamento também foi um dos fatores considerados para a sua escolha.

Os pontos amostrais P4 a P7 localizaram-se em áreas utilizadas principalmente para plantações de cana de açúcar. Nestes pontos a mata ciliar se encontrou mais bem preservada do que nos demais pontos estudados.

Geologicamente, a microbacia do rio Cabeça é composta por rochas sedimentares e vulcânicas das eras Paleozóicas (Figura 3): Grupo Passa Dois (Formação Corumbataí nos pontos P3 e P4 e Formação Irati nos ponto P5, P6 e P7); Mesozoicas: Grupo São Bento (Formação Piramboia nos pontos P1, P2 e P3 além das Formações Botucatu e Serra Geral, estas mais próximas do ponto P1) e Cenozoica: Formação Rio Claro e depósitos recentes nos pontos P3 e P4.

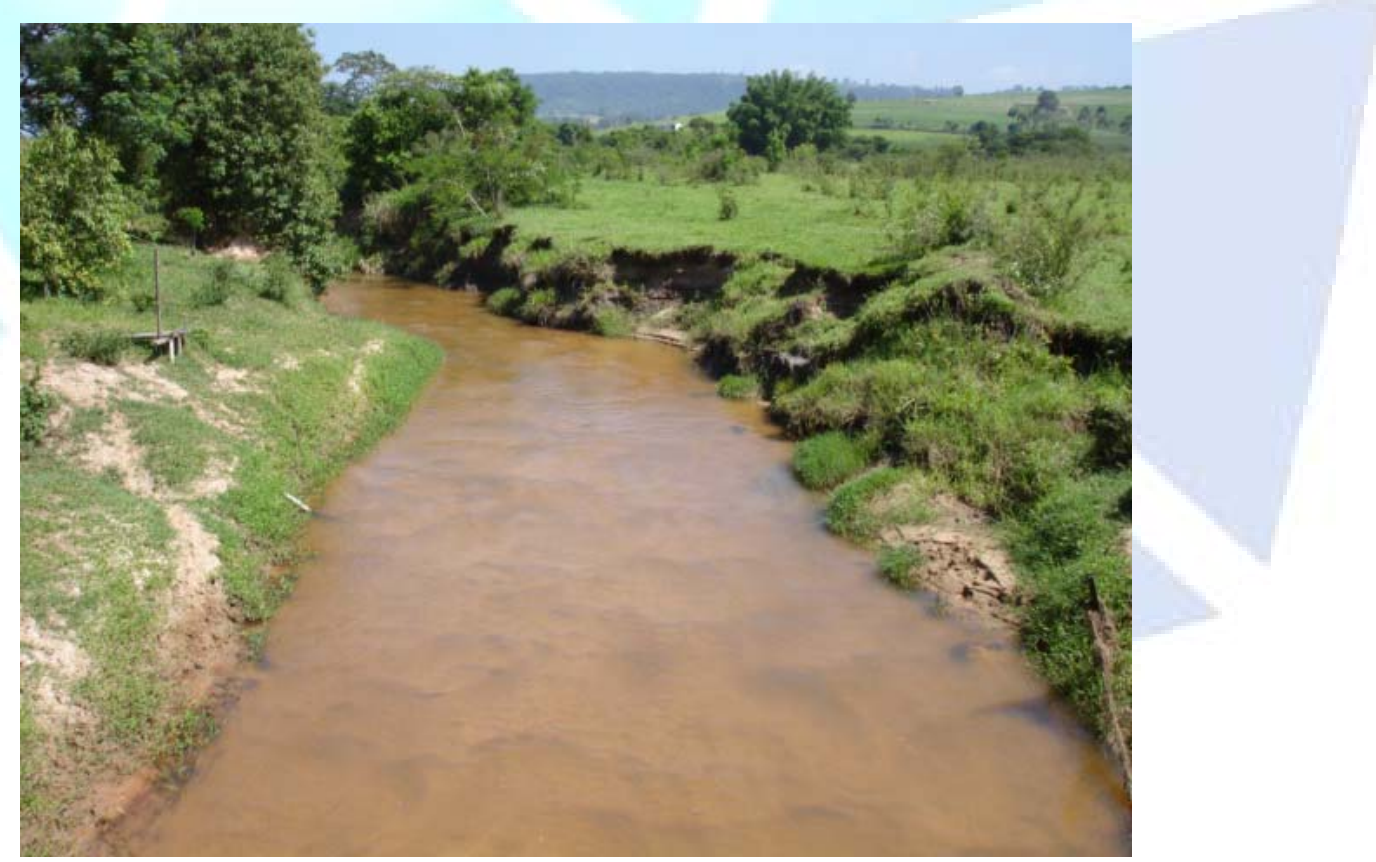

Figura 2. Ponto de amostragem P2 no Rio Cabeça indicando a ausência de mata ciliar e assoreamento.

$\mathrm{Na}$ maior parte da microbacia há predomínio de solos podzólico avermelhado amarelo, predominando nas áreas adjacentes aos pontos de amostragem, seguido de latossolo vermelho escuro. Merece destaque também o solo litólico que tem ocorrência próxima a P1 (Figura 4). O clima é do tipo Cwa (w: seca no inverno, a: mês mais quente com temperatura superior a $22^{\circ} \mathrm{C}$ ). A 
área pode ser enquadrada dentro de um clima tropical com duas estações bem definidas, alternadamente seca e úmida (MONTEIRO, 1973).

\subsection{Procedimento analítico}

As coletas foram realizadas mensalmente durante o período de abril de 2007 a março de 2009, totalizando 24 amostragens. O período estudado engloba as estações seca (abril a setembro) e chuvosa (outubro a março). Para a coleta e transporte das amostras seguiram-se os procedimentos estabelecidos pelo Guia de Coleta e Preservação de Amostras de Água da CETESB (CETESB, 1987).

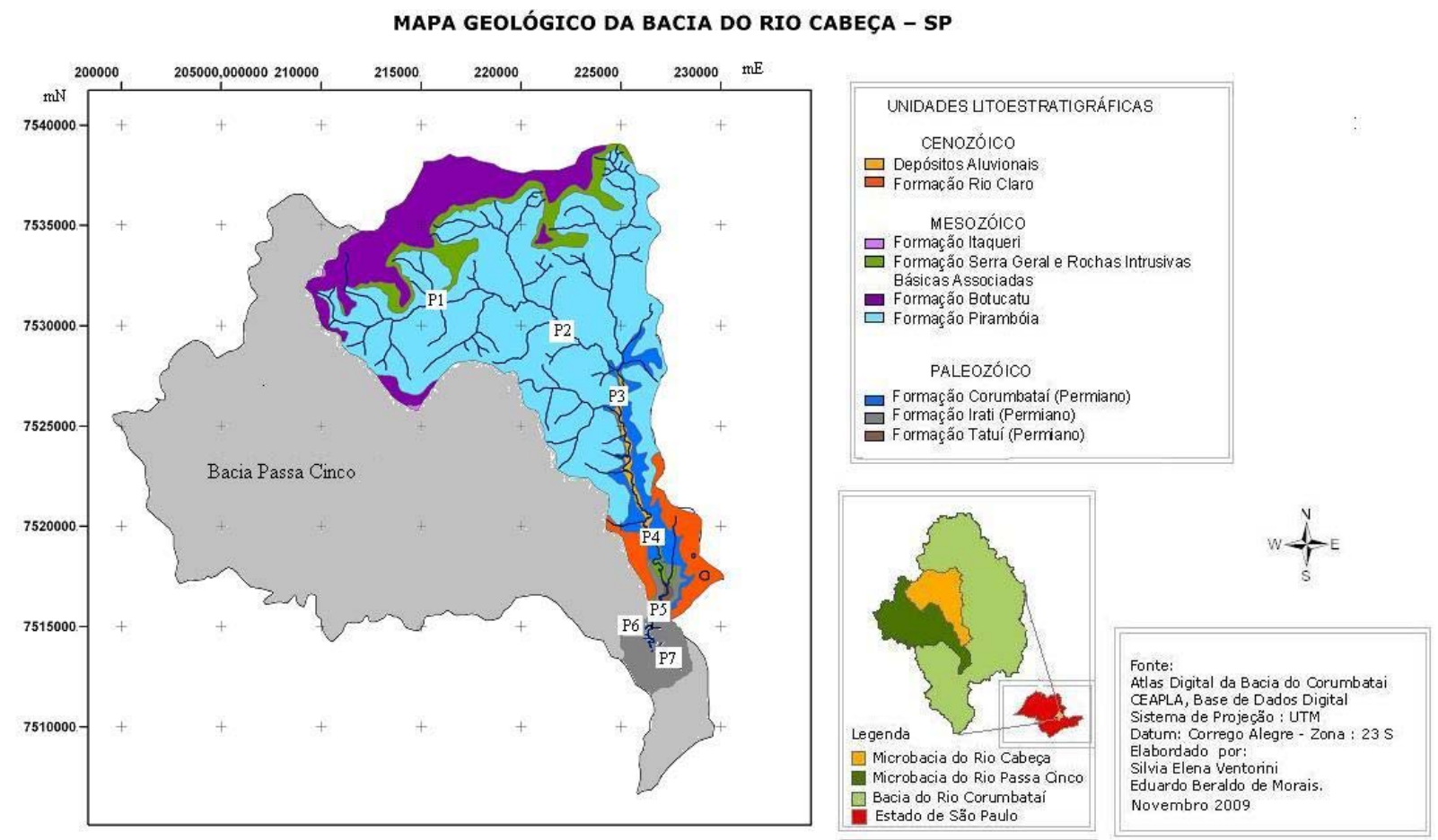

$\begin{array}{lll}2,5 & 0 & \mathrm{Km}\end{array}$

Figura 3. Unidades litoestratigráficas na microbacia do rio cabeça. Fonte: Morais (2010). 


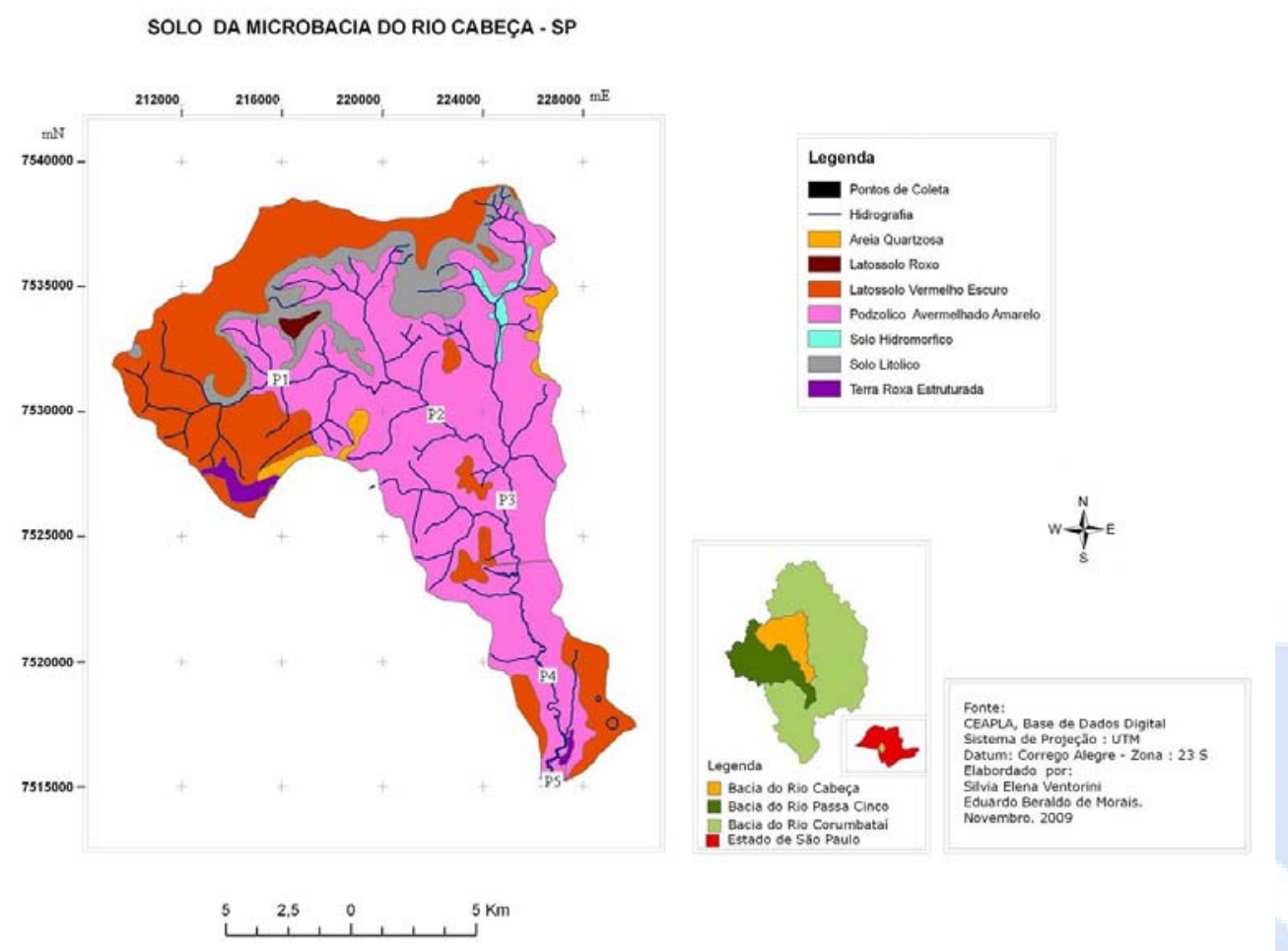

Figura 4. Mapa pedológico da microbacia do rio Cabeça, SP. Fonte: Morais (2010).

No laboratório, as amostras de água foram filtradas em sistema a vácuo usando filtro MGC, $47 \mathrm{~mm}$ de diâmetro e $0,45 \mu \mathrm{m}$ de porosidade e posteriormente, acidificadas com ácido nítrico concentrado acarretando uma concentração final de $2 \%(\mathrm{v} / \mathrm{v})$. Os metais determinados foram: Al, $\mathrm{Cd}, \mathrm{Cr}, \mathrm{Cu}, \mathrm{Fe}, \mathrm{Mn}, \mathrm{Ni}, \mathrm{Pb}, \mathrm{Zn}, \mathrm{Ca}$ e $\mathrm{Mg}$. A leitura foi efetuada em Espectrômetro de Emissão Óptica Sequencial com Plasma Acoplado Indutivamente (ICP OES) marca GBC, modelo integra XL de acordo com a metodologia descrita no Standard Methods for the Examination of Water and Wastewater (APHA, 1998). As condições de operação do equipamento se encontram na Tabela 2.

Tabela 2 - Condições de operação do Espectrômetro de Emissão Óptica Sequencial com Plasma Acoplado

\begin{tabular}{|c|c|c|}
\hline \multicolumn{3}{|l|}{ ICP OES } \\
\hline Potência incidente (W) & & 200 \\
\hline Vazão do gás plasma (L. min $^{-1}$ ) & & 10,0 \\
\hline Vazão do gás auxiliar ( $\left.\mathrm{Lmin}^{-1}\right)$ & & 0,5 \\
\hline Vazão do gás Nebulizador $\left(\operatorname{Lmin}^{-1}\right)$ & & 0,5 \\
\hline Altura de Observação (mm) & & 10,0 \\
\hline Elemento & $\begin{array}{l}\text { Linhas de } \\
\text { emissão (nm) }\end{array}$ & $\begin{array}{c}\text { Limites de } \\
\text { detecção }\left(\mathrm{mg} . \mathrm{L}^{-1}\right)\end{array}$ \\
\hline $\mathrm{Al}$ & 167,081 & 0,009 \\
\hline $\mathrm{Cd}$ & 228,802 & 0,004 \\
\hline $\mathrm{Cu}$ & 324,754 & 0,011 \\
\hline $\mathrm{Fe}$ & 259,940 & 0,004 \\
\hline $\mathrm{Mg}$ & 285,213 & 0,007 \\
\hline $\mathrm{Mn}$ & 257,610 & 0,0008 \\
\hline $\mathrm{Pb}$ & 220,353 & 0,030 \\
\hline $\mathrm{Zn}$ & 213,856 & 0,003 \\
\hline $\mathrm{Ca}$ & 422,673 & 0,014 \\
\hline $\mathrm{Cr}$ & 283,563 & 0,028 \\
\hline $\mathrm{Ni}$ & 231,304 & 0,015 \\
\hline
\end{tabular}




\subsection{Análise estatística e interpretação dos dados obtidos}

Com o intuito de identificar os possíveis fatores/fontes determinantes dos fatores avaliados no sistema aquático nos períodos seco (abril a setembro) e chuvoso (outubro a março), optou-se pela aplicação da técnica estatística multivariada por meio da Análise de Componentes Principais (ACP). Os procedimentos multivariados, k-médias e componentes principais foram aplicados aos referidos metais com valores padronizados, resultando em média nula e variância unitária (HARTINGAN, 1975). As análises estatísticas foram realizadas no software STATISTICA versão 7.1 (STATSOFT, 2005).

\section{RESULTADOS E DISCUSSÃO}

Muitos dos metais aqui analisados são fisiologicamente essenciais para animais e plantas: $\mathrm{Fe}$ (constituinte da molécula, dentre outras, de hemoglobina e ferrodoxina, esta mediadora da transferência de elétrons em diversas reações metabólicas); Zn (cofator enzimático e constituinte de várias moléculas que integram as membranas celulares, particularmente os leucócitos); $\mathrm{Mg}$ (cofator enzimático e integrante da molécula de clorofila); $\mathrm{Cu}$ (biossíntese de colágeno e elastina, formação da melanina); $\mathrm{Ni}$ e $\mathrm{Mn}$ (cofatores enzimáticos); $\mathrm{Cr}$ (importante para o metabolismo dos carboidratos) (BERG et al., 2010). Outros metais, entretanto, não possuem funções fisiológicas conhecidas nos organismos e são potencialmente tóxicos como é o caso do $\mathrm{Cd}$ e $\mathrm{Pb}$. Mesmo aqueles metais essenciais ao metabolismo dos organismos, geralmente pequenas quantidades são requeridas sendo que em maiores concentrações efeitos tóxicos podem ser observados.

Nas amostras analisadas neste estudo, o $\mathrm{Cd}$ foi detectado somente na estação seca do ano de 2007 nos pontos P2 a P7 em julho, P2 e P4 em agosto e P2 a P7 em setembro (Tabela 3). O Pb foi detectado no início da estação seca em 2008 nos pontos P1, P4, P6 e P7 em maio e P2, P3 e P4 em junho e também na estação chuvosa em 2009 nos pontos P1, P2, P3, P6 e P7 em janeiro e março (Tabela 4). Os valores destes dois elementos ficaram abaixo do limite máximo estabelecido pelo Decreto Estadual 8468/1976 (SÃO PAULO, 1976) para rios de classe 2, que fixou como padrão os valores de $0,01 \mathrm{mg} . \mathrm{L}^{-1}$ para o $\mathrm{Cd}$ e $0,1 \mathrm{mg} . \mathrm{L}^{-1}$ para o $\mathrm{Pb}$. A comparação destes resultados com os limites estabelecidos pela Resolução CONAMA 357/2005 (BRASIL, 2005), entretanto, mostrou que os valores foram superiores ao limite máximo estabelecido pela legislação federal já que esta é mais restritiva para estes dois metais fixando os limites máximos de $0,001 \mathrm{mg} . \mathrm{L}^{-1}$ para o Cd e 0,01 $\mathrm{mg} . \mathrm{L}^{-1}$ para o $\mathrm{Pb}$. As principais fontes destes dois elementos no ambiente são principalmente os efluentes de indústrias de baterias, galvanoplastia e plásticos (PANDEY et al., 2008), aterros sanitários (HE et al., 2006) e fertilizantes fosfatados (RAMALHO et al., 1999; MENDES et al., 2006). Wu et al. (2012) também destacaram a aplicação de dejetos de animais em solos, especialmente suínos, como uma fonte de poluição por Cd e outros elementos tóxicos já que os metais são amplamente utilizados como aditivos na alimentação animal. Dessa forma, os valores de $\mathrm{Cd}$ e $\mathrm{Pb}$ encontrados podem estar relacionados com as atividades agropecuárias desenvolvidas na área de estudo.

Tabela 3 - Concentrações de Cd (mg. $\left.\mathrm{L}^{-1}\right)$ encontradas em algumas amostras de água do rio Cabeça (P1 a P5) e rio Passa Cinco (P6 e P7) durante o período de amostragem (abril de 2007 a março de 2009).

\begin{tabular}{|c|c|c|c|c|c|c|c|}
\hline Meses & P1 & P2 & P3 & P4 & P5 & P6 & P7 \\
\hline • & - & - & - & . & . & . & . \\
\hline $\mathbf{J u l} / \mathbf{0 7}$ & . & $0,008 \pm 5,2 \mathrm{e}-4$ & $0,007 \pm 5,1 \mathrm{e}-4$ & $0,007 \pm 2,0 \mathrm{e}-3$ & $0,007 \pm 9,2 \mathrm{e}-4$ & $0,008 \pm 4,9 \mathrm{e}-4$ & $0,007 \pm 1,9 \mathrm{e}-3$ \\
\hline Ago/07 & . & $0,005 \pm 6,8 \mathrm{e}-4$ & . & $0,004 \pm 1,6 \mathrm{e}-3$ & . & . & . \\
\hline Set/07 & - & $0,006 \pm 9,2 \mathrm{e}-4$ & $0,004 \pm 3,8 \mathrm{e}-4$ & $0,006 \pm 9,3 \mathrm{e}-4$ & $0,004 \pm 5,5 \mathrm{e}-4$ & $0,006 \pm 1,2 \mathrm{e}-3$ & $0,004 \pm 6,4 \mathrm{e}-4$ \\
\hline . & . & . & - & . & . & . & . \\
\hline
\end{tabular}

Legenda: $(\cdot)$ = não detectado. Os dados são médias de três leituras da amostra. 
Tabela 4 - Concentrações de $\mathrm{Pb}\left(\mathrm{mg} \cdot \mathrm{L}^{-1}\right)$ encontradas em algumas amostras de água do rio Cabeça (P1 a P5) e rio Passa Cinco (P6 e P7) durante o período de amostragem (abril de 2007 a março de 2009).

\begin{tabular}{|c|c|c|c|c|c|c|c|}
\hline Meses & P1 & $\mathbf{P 2}$ & $\mathbf{P 3}$ & P4 & P5 & P6 & P7 \\
\hline • & - & - & • & • & - & - & - \\
\hline Mai/08 & $0,03 \pm 1,8 \mathrm{e}-2$ & . & . & $0,04 \pm 9,8 \mathrm{e}-3$ & . & $0,03 \pm 1,5 \mathrm{e}-2$ & $0,05 \pm 1,2 \mathrm{e}-2$ \\
\hline Jun/08 & . & $0,05 \pm 2,3 \mathrm{e}-2$ & $0,04 \pm 1,3 \mathrm{e}-2$ & $0,04 \pm 1,3 \mathrm{e}-2$ & . & . & . \\
\hline • & • & • & . & . & $\cdot$ & • & $\cdot$ \\
\hline • & • & - & 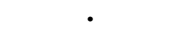 & • & - & • & - \\
\hline Jan/09 & $0,04 \pm 1,5 \mathrm{e}-2$ & $0,04 \pm 1,9 \mathrm{e}-2$ & $0,03 \pm 9,6 \mathrm{e}-3$ & . & . & $0,05 \pm 2,8 \mathrm{e}-2$ & $0,05 \pm 1,6 \mathrm{e}-2$ \\
\hline · & · & • & . & . & . & . & . \\
\hline Mar/09 & $0,03 \pm 1,1 \mathrm{e}-2$ & $0,04 \pm 2,1 \mathrm{e}-2$ & $0,04 \pm 1,3 e-2$ & . & . & $0,03 \pm 1,5 \mathrm{e}-2$ & $0,03 \pm 1,8 \mathrm{e}-2$ \\
\hline
\end{tabular}

Legenda: $(\cdot)$ = não detectado. Os dados são médias de três leituras da amostra

Os metais $\mathrm{Cr}$, $\mathrm{Cu}$ e Ni não foram detectados nos pontos amostrados, provavelmente estando em concentrações menores àquelas possíveis de serem detectadas pelas metodologias utilizadas e sensibilidade do equipamento (Limites de detecção: $\mathrm{Cr}=0,028 \mathrm{mg} . \mathrm{L}^{-1} ; \mathrm{Cu}=0,011 \mathrm{mg} \cdot \mathrm{L}^{-1} ; \mathrm{Ni}=$ 0,015 mg. $\mathrm{L}^{-1}$ ). Os demais metais, Fe, $\mathrm{Al}, \mathrm{Mn}, \mathrm{Zn}, \mathrm{Ca}$ e $\mathrm{Mg}$, foram detectados em todos os pontos amostrados (Tabelas 5 a 10).

Tabela 5 - Variação espaço-temporal de $\mathrm{Fe}\left(\mathrm{mg} . \mathrm{L}^{-1}\right)$ determinada no período de abril de 2007 a março de 2009 nos pontos de amostragem.

\begin{tabular}{|c|c|c|c|c|c|c|c|}
\hline Período & P1 & P2 & P3 & P4 & P5 & P6 & P7 \\
\hline Abr/07 & $74 \pm 7,2 \mathrm{e}-3$ & $89 \pm 4,2 \mathrm{e}-3$ & $10 \pm 8,4 \mathrm{e}-3$ & $, 15 \pm 1,4 \mathrm{e}-2$ & $22 \pm 1,7 \mathrm{e}-2$ & $0,80 \pm 8,7 \mathrm{e}-3$ & $0,96 \pm 7,6 \mathrm{e}-3$ \\
\hline & $53 \pm 3,8 \mathrm{e}-3$ & $59 \pm 3,7 e-3$ & $30 \pm 3,2 \mathrm{e}-3$ & e-3 & $2 \pm 7,8 \mathrm{e}-3$ & -3 & $0,72 \pm 3,2 \mathrm{e}-3$ \\
\hline Jun/07 & $5 \pm 1,8 \mathrm{e}-3$ & $4 \pm 6$ & $60 \pm 5$ & -3 & $62 \pm 4,3 e-3$ & $e-3$ & $0,52 \pm$ \\
\hline & $7 \pm 5,5 \mathrm{e}-3$ & $0,63 \pm 1,1 \mathrm{e}-2$ & $32 \pm 2,7 \mathrm{e}-3$ & -3 & $0 \pm 4, \varepsilon$ & & $7 e-3$ \\
\hline & $71 \pm 5,1 \mathrm{e}-3$ & $1 \mathrm{e}-2$ & $72 \pm 1,1 \mathrm{e}-2$ & $0,84 \pm 1,5 \mathrm{e}-2$ & $0,75 \pm 4,6 \mathrm{e}-4$ & $0,46 \pm$ & $0,58 \pm 1,3 \mathrm{e}-3$ \\
\hline & $1,02 \pm 1,6 \mathrm{e}-2$ & $0,76 \pm 8,3 \mathrm{e}-3$ & $0,86 \pm 8,7 \mathrm{e}-3$ & -3 & $0,83 \pm 1,5 \mathrm{e}-2$ & -3 & $\pm 4,5 \mathrm{e}-3$ \\
\hline Out/07 & $1 \pm 9,6 \mathrm{e}-3$ & $9 \pm 4,0 \mathrm{e}-3$ & $1,11 \pm 3,2 \mathrm{e}-3$ & $1,25 \pm$ & $1,04 \pm 1,5 \mathrm{e}-2$ & $0,74 \pm$ & $0,89 \pm 4,2 \mathrm{e}-3$ \\
\hline & $0,92 \pm 2,3 e-3$ & $4 \pm 4,4 \mathrm{e}-3$ & $1,01 \pm 2,1$ & 1 , & $1,08 \pm 8$ & e-3 & $0,94 \pm$ \\
\hline & $1 \pm 1,5 \mathrm{e}-3$ & $6 \pm 1,5 \mathrm{e}-2$ & $1,26 \pm 1$ & -2 & $1,29 \pm 4$ & & $0,76 \pm$ \\
\hline & $0,81 \pm 1,7 \mathrm{e}-2$ & $0,90 \pm 1$ & $1,22 \pm 1,3 \mathrm{e}-2$ & 1,4 & $1,29 \pm 1,5$ & & $1,11 \pm$ \\
\hline & $2 \pm 7,3 \mathrm{e}-3$ & $e-3$ & \pm 1 & -2 & $1,40 \pm 2,1 \mathrm{e}-2$ & & $9 e-3$ \\
\hline & $0,83 \pm 7,6 \mathrm{e}-3$ & $\pm 1,0 \mathrm{e}-2$ & $1,08 \pm 5$ & $1,18 \pm$ & $1,23 \pm 3,9 \mathrm{e}-3$ & & $0,96 \pm$ \\
\hline & $8 \pm 1,2 \mathrm{e}-2$ & $\pm 3,3 \mathrm{e}-3$ & e-3 & -2 & $1,29 \pm 1,8 \mathrm{e}-2$ & $e-4$ & $0,94 \pm$ \\
\hline & $0,46 \pm 8,1 \mathrm{e}-3$ & $e-3$ & $0,68 \pm 6$ & & $0,86 \pm 8,1 \mathrm{e}-3$ & 0,46 & $0,64 \pm$ \\
\hline $\mathbf{J u}$ & $3 \pm 6,3 \mathrm{e}-4$ & e-3 & \pm 1 & $e-3$ & $0,64 \pm 1,4 \mathrm{e}-2$ & $0,45 \pm$ & $0,52 \pm 1,2 \mathrm{e}-3$ \\
\hline & $0,39 \pm 1,2 \mathrm{e}-2$ & $0,34 \pm 9,1 \mathrm{e}-3$ & $0,41 \pm 7,9 \mathrm{e}-3$ & & $0,49 \pm 5,4 \mathrm{e}-3$ & $0,33 \pm 4$ & $0,42 \pm 1,5 \mathrm{e}-3$ \\
\hline Ag & $6 \pm 7,4 \mathrm{e}-3$ & $2 e-3$ & $0,54 \pm 7$ & $\mathrm{e}-2$ & $0,66 \pm 7,8 \mathrm{e}-3$ & $0,48 \pm 2,2 \mathrm{e}-3$ & $0,56 \pm 4,8 \mathrm{e}-3$ \\
\hline & & & & & $1,31 \pm 1,8$ & $0,69 \pm 1,2 \mathrm{e}-2$ & $1,01 \pm 2,7 \mathrm{e}-2$ \\
\hline Ou & $0 \pm 7$ & e-3 & $2 \pm 1$ & $0,81 \pm 5,1 \mathrm{e}-3$ & $0,73 \pm 7,1 \mathrm{e}-3$ & $e-3$ & $0,63 \pm 6,9 \mathrm{e}-3$ \\
\hline Nov/08 & $3 \pm 5,9 \mathrm{e}-3$ & & $0,90 \pm 7,5 \mathrm{e}-3$ & $1,19 \pm$ & $1,23 \pm 3,3 \mathrm{e}-2$ & & $1,03 \pm 1,1 \mathrm{e}-2$ \\
\hline Dez/08 & $0,86 \pm 6,6 \mathrm{e}-3$ & $9 e-3$ & $1,34 \pm 4,7 \mathrm{e}-3$ & $3 \pm 3$ & $1,22 \pm 4,2 \mathrm{e}-3$ & $0,95 \pm 4,3 \mathrm{e}-3$ & $1,43 \pm 6,2 \mathrm{e}-3$ \\
\hline & $3 \pm 6,1 \mathrm{e}-3$ & $1,20 \pm 1$ & $1,82 \pm 4$ & $1,96 \pm 6$ & $1,81 \pm 1,9 \mathrm{e}-2$ & $1,10 \pm 3$ & $1,29 \pm 7,1 \mathrm{e}-3$ \\
\hline Fev/09 & $1,09 \pm 4,9 \mathrm{e}-3$ & $1,31 \pm 5,9 \mathrm{e}-3$ & $1,91 \pm 7,3 \mathrm{e}-3$ & $1,93 \pm 8,9 \mathrm{e}-3$ & $1,90 \pm 2,1 \mathrm{e}-2$ & $1,25 \pm 1,6 \mathrm{e}-2$ & $1,46 \pm 1,2 \mathrm{e}-2$ \\
\hline Mar/09 & $1,16 \pm 7,5 \mathrm{e}-3$ & $1,36 \pm 6,9 \mathrm{e}-3$ & $1,80 \pm 8,9 \mathrm{e}-3$ & $1,82 \pm 1,0 \mathrm{e}-2$ & $1,97 \pm 1,7 \mathrm{e}-2$ & $1,17 \pm 1,9 \mathrm{e}-2$ & $1,55 \pm 1,6 \mathrm{e}-2$ \\
\hline
\end{tabular}

Os dados são médias de três leituras da amostra. 
Tabela 6 - Variação espaço-temporal de $\mathrm{Al}\left(\mathrm{mg} \cdot \mathrm{L}^{-1}\right)$ determinada no período de abril de 2007 a março de 2009 nos pontos de amostragem.

\begin{tabular}{|c|c|c|c|c|c|c|c|}
\hline Período & P1 & P2 & P3 & P4 & P5 & P6 & P7 \\
\hline Abr/07 & $0,12 \pm 1,8 \mathrm{e}-3$ & $0,19 \pm 2,0 \mathrm{e}-3$ & $0,21 \pm 2,2 \mathrm{e}-3$ & $0,27 \pm 5,7 \mathrm{e}-3$ & $0,33 \pm 3,1 \mathrm{e}-3$ & $0,24 \pm 2,9 \mathrm{e}-3$ & $0,27 \pm 5,8 \mathrm{e}-3$ \\
\hline Mai/07 & $0,21 \pm 3,2 \mathrm{e}-3$ & $0,16 \pm 1,7 \mathrm{e}-3$ & $0,20 \pm 4,0 \mathrm{e}-3$ & $0,25 \pm 3,1 \mathrm{e}-2$ & $0,29 \pm 5,3-3$ & $0,21 \pm 7,0 \mathrm{e}-3$ & $0,22 \pm 4,3 \mathrm{e}-3$ \\
\hline Jun/07 & $0,11 \pm 2,2 \mathrm{e}-3$ & $0,12 \pm 3,1 \mathrm{e}-3$ & $0,13 \pm 1,2 \mathrm{e}-3$ & $0,20 \pm 4,8 \mathrm{e}-3$ & $0,17 \pm 4,6 \mathrm{e}-3$ & $0,09 \pm 2,5 \mathrm{e}-3$ & $0,13 \pm 2,0 \mathrm{e}-3$ \\
\hline Jul/07 & $0,11 \pm 1,2 \mathrm{e}-3$ & $0,20 \pm 8,0 \mathrm{e}-3$ & $0,31 \pm 7,2 \mathrm{e}-3$ & $0,26 \pm 1,0 \mathrm{e}-2$ & $0,23 \pm 6,0 \mathrm{e}-3$ & $0,33 \pm 3,0 \mathrm{e}-4$ & $0,40 \pm 1,1 \mathrm{e}-2$ \\
\hline Ago/07 & $0,06 \pm 4,5 \mathrm{e}-3$ & $0,12 \pm 6,8 \mathrm{e}-3$ & $0,14 \pm 3,7 \mathrm{e}-3$ & $0,15 \pm 3,8 \mathrm{e}-3$ & $0,14 \pm 3,9 \mathrm{e}-3$ & $0,11 \pm 3,0 \mathrm{e}-3$ & $0,11 \pm 2,2 \mathrm{e}-3$ \\
\hline Set/07 & $0,07 \pm 3,9 \mathrm{e}-3$ & $0,10 \pm 2,5 \mathrm{e}-3$ & $0,18 \pm 9,8 \mathrm{e}-3$ & $0,16 \pm 1,4 \mathrm{e}-3$ & $0,19 \pm 5,2 \mathrm{e}-3$ & $0,07 \pm 2,0 \mathrm{e}-3$ & $0,11 \pm 6,3 \mathrm{e}-3$ \\
\hline Out/07 & $0,06 \pm 2,2 \mathrm{e}-3$ & $0,20 \pm 1,2 \mathrm{e}-2$ & $0,21 \pm 6,7 \mathrm{e}-3$ & $0,38 \pm 9,9 \mathrm{e}-3$ & $0,31 \pm 1,1 \mathrm{e}-2$ & $0,14 \pm 1,1 \mathrm{e}-2$ & $0,32 \pm 3,3 \mathrm{e}-3$ \\
\hline Nov/07 & $0,05 \pm 1,6 \mathrm{e}-3$ & $0,12 \pm 2,4 \mathrm{e}-3$ & $0,15 \pm 6,4 \mathrm{e}-3$ & $0,21 \pm 5,0 \mathrm{e}-3$ & $0,21 \pm 1,1 \mathrm{e}-2$ & $0,08 \pm 1,8 \mathrm{e}-3$ & $0,10 \pm 1,5 \mathrm{e}-3$ \\
\hline Dez/07 & $1,34 \pm 1,9 \mathrm{e}-2$ & $0,39 \pm 2,3 \mathrm{e}-2$ & $0,42 \pm 1,7 \mathrm{e}-2$ & $1,79 \pm 2,9 \mathrm{e}-2$ & $2,19 \pm 8,9 \mathrm{e}-2$ & $0,98 \pm 6,6 \mathrm{e}-2$ & $1,47 \pm 2,3 \mathrm{e}-2$ \\
\hline Jan/08 & $0,11 \pm 4,6 \mathrm{e}-3$ & $0,30 \pm 7,0 \mathrm{e}-3$ & $0,26 \pm 1,9 \mathrm{e}-2$ & $0,48 \pm 1,5 \mathrm{e}-2$ & $0,35 \pm 6$ & $0,39 \pm 4,9 \mathrm{e}-3$ & $0,53 \pm 1,3 \mathrm{e}-2$ \\
\hline Fev/08 & $0,36 \pm 2,4 \mathrm{e}-3$ & $0,71 \pm 3,6 \mathrm{e}-2$ & $0,78 \pm 1,4 \mathrm{e}-2$ & $0,56 \pm 1,4 \mathrm{e}-2$ & $0,49 \pm 4,3 e-3$ & $0,89 \pm 2,1 \mathrm{e}-2$ & $0,87 \pm 8,5 \mathrm{e}-3$ \\
\hline Mar/08 & $0,10 \pm 6,2 \mathrm{e}-3$ & $0,20 \pm 7,0 \mathrm{e}-4$ & $0,05 \pm 4,0 \mathrm{e}-3$ & $0,16 \pm 1,1 \mathrm{e}-2$ & $7 \pm 4,5 \mathrm{e}-3$ & $0,09 \pm 2,4 \mathrm{e}-3$ & $0,10 \pm 3,3 \mathrm{e}-3$ \\
\hline Abr/08 & $0,03 \pm 2,2 \mathrm{e}-3$ & $0,06 \pm 9,1 \mathrm{e}-4$ & $0,22 \pm 2,7 \mathrm{e}-3$ & $0,11 \pm 3,4 \mathrm{e}-3$ & $0,24 \pm 3,4 \mathrm{e}-3$ & $0,08 \pm 1,3 \mathrm{e}-3$ & $0,14 \pm 7,6 \mathrm{e}-3$ \\
\hline Mai/08 & $0,08 \pm 8,7 \mathrm{e}-3$ & $0,12 \pm 1,4 \mathrm{e}-3$ & $0,11 \pm 8,9 \mathrm{e}-3$ & $0,26 \pm 5,0 \mathrm{e}-3$ & $0,20 \pm 9,9 \mathrm{e}-3$ & $0,16 \pm 1,8 \mathrm{e}-3$ & $0,17 \pm 2,0 \mathrm{e}-3$ \\
\hline Jun/08 & $0,07 \pm 2,3 e-3$ & $0,13 \pm 7,4 \mathrm{e}-3$ & $0,13 \pm 8,7 \mathrm{e}-3$ & $0,13 \pm 5,7 e-3$ & $0,15 \pm 1,4 \mathrm{e}-2$ & $0,09 \pm 7,2 \mathrm{e}-3$ & $0,12 \pm 5,2 \mathrm{e}-3$ \\
\hline Jul/08 & $0,06 \pm 1,7 \mathrm{e}-3$ & $0,08 \pm 7,7 \mathrm{e}-3$ & $0,10 \pm 1,4 \mathrm{e}-3$ & $0,19 \pm 9,7 \mathrm{e}-3$ & $0,22 \pm 5,5 \mathrm{e}-3$ & $0,09 \pm 5,8 \mathrm{e}-3$ & $0,20 \pm 1,1 \mathrm{e}-2$ \\
\hline Ago/08 & $0,04 \pm 4,9 \mathrm{e}-3$ & $0,08 \pm 3,4 \mathrm{e}-3$ & $0,09 \pm 4,7 \mathrm{e}-3$ & $0,14 \pm 7,4 \mathrm{e}-3$ & $0,19 \pm 4,7 \mathrm{e}-3$ & $0,08 \pm 1,3 e-3$ & $0,12 \pm 7,1 \mathrm{e}-3$ \\
\hline Set/08 & $0,17 \pm 7,5 \mathrm{e}-3$ & $0,33 \pm 1,6 \mathrm{e}-2$ & $0,36 \pm 5,6 \mathrm{e}-3$ & $0,88 \pm 2,1 \mathrm{e}-2$ & $1,15 \pm 3,5 \mathrm{e}-2$ & $0,42 \pm 1,7 \mathrm{e}-2$ & $0,81 \pm 2,0 \mathrm{e}-3$ \\
\hline Out/08 & $0,05 \pm 3,8 \mathrm{e}-3$ & $0,07 \pm 2,5 \mathrm{e}-3$ & $0,10 \pm 9,2 \mathrm{e}-3$ & $0,10 \pm 5,8 \mathrm{e}-3$ & $0,11 \pm 5,3 e-3$ & $0,06 \pm 3,9 \mathrm{e}-3$ & $0,06 \pm 7,1 \mathrm{e}-3$ \\
\hline Nov/08 & $0,01 \pm 7,3 e-3$ & $0,14 \pm 9,1 \mathrm{e}-3$ & $0,16 \pm 1,6 \mathrm{e}-2$ & $0,53 \pm 1,9 e-2$ & $0,81 \pm 7,7 \mathrm{e}-3$ & $0,46 \pm 1,2 \mathrm{e}-2$ & $0,78 \pm 1,7 \mathrm{e}-2$ \\
\hline Dez/08 & $0,18 \pm 6,5 \mathrm{e}-3$ & $0,42 \pm 7,5 \mathrm{e}-3$ & $0,17 \pm 8,9 \mathrm{e}-3$ & $0,39 \pm 7,9 \mathrm{e}-3$ & $0,38 \pm 8,3 \mathrm{e}-3$ & $0,40 \pm 7,2 \mathrm{e}-3$ & $1,19 \pm 6,9 \mathrm{e}-3$ \\
\hline Jan/09 & $0,07 \pm 4,2 \mathrm{e}-3$ & $0,11 \pm 1,2 \mathrm{e}-3$ & $0,18 \pm 3,7 \mathrm{e}-3$ & $0,31 \pm 4,7 \mathrm{e}-3$ & $0,35 \pm 5,3 \mathrm{e}-3$ & $0,28 \pm 5,1 \mathrm{e}-3$ & $0,26 \pm 6,3 \mathrm{e}-3$ \\
\hline Fev/09 & $0,08 \pm 5,3 e-3$ & $0,20 \pm 5,9 \mathrm{e}-3$ & $0,17 \pm 6,3 \mathrm{e}-3$ & $0,32 \pm 4,4 \mathrm{e}-2$ & $0,40 \pm 2,7 \mathrm{e}-2$ & $0,31 \pm 4,2 \mathrm{e}-2$ & $0,52 \pm 3,2 \mathrm{e}-3$ \\
\hline Mar/09 & $0,09 \pm 5,1 \mathrm{e}-3$ & $0,26 \pm 7,2 \mathrm{e}-3$ & $0,27 \pm 4,4 \mathrm{e}-3$ & $0,28 \pm 3,8 \mathrm{e}-3$ & $0,34 \pm 5,4 \mathrm{e}-3$ & $0,34 \pm 6,3 \mathrm{e}-3$ & $0,33 \pm 6,1 \mathrm{e}-3$ \\
\hline
\end{tabular}

Os dados são médias de três leituras da amostra.

Tabela 7 - Variação espaço-temporal de $\mathrm{Zn}\left(\mathrm{mg} \cdot \mathrm{L}^{-1}\right)$ determinada no período de abril de 2007 a março de 2009 nos pontos de amostragem.

\begin{tabular}{|c|c|c|c|c|c|c|c|}
\hline Período & P1 & $\mathbf{P 2}$ & P3 & P4 & P5 & P6 & P7 \\
\hline Abr/07 & $0,051 \pm 3,2 \mathrm{e}-4$ & $052 \pm 2,8 \mathrm{e}-4$ & $042 \pm 4,4 \mathrm{e}-4$ & $0,048 \pm 5,6 \mathrm{e}-4$ & $0,042 \pm 3,3 \mathrm{e}-4$ & $0,042 \pm 6,2 \mathrm{e}-4$ & $0,040 \pm 4,3 \mathrm{e}-4$ \\
\hline Mai/07 & $0,048 \pm 2,4 \mathrm{e}-4$ & $043 \pm 5,2 \mathrm{e}-4$ & $043 \pm 7,1 \mathrm{e}-4$ &, $5 e-4$ & $044 \pm 3,1 \mathrm{e}-4$ & $64 \pm 2,1 \mathrm{e}-3$ & $0,044 \pm 1,2 \mathrm{e}-3$ \\
\hline Jun/07 & $0,042 \pm 3,1 \mathrm{e}-4$ & $0,040 \pm 3,3 e-4$ & $0,038 \pm 4,7 e-4$ & $0,037 \pm 2,9 \mathrm{e}-4$ & $0,038 \pm 6,7 \mathrm{e}-4$ & $0,034 \pm 1,7 \mathrm{e}-4$ & $0,032 \pm 2,6 \mathrm{e}-4$ \\
\hline Jul/07 & $0,039 \pm$ & $0,036 \pm 6$ & $0,041 \pm 1,1 \mathrm{e}-3$ & $038 \pm$ & $033 \pm 3,8 \mathrm{e}-4$ & $40 \pm$ & $9 e-5$ \\
\hline Ago/07 & $0,034 \pm 2,5 \mathrm{e}-4$ & $0,038 \pm 8,9 \mathrm{e}-4$ & $0,035 \pm 1,2 \mathrm{e}-3$ & $0,040 \pm 9,2 \mathrm{e}-3$ & $0,032 \pm 5,2 \mathrm{e}-4$ & $0,033 \pm 9,7 \mathrm{e}-4$ & $0,028 \pm 5,3 \mathrm{e}-4$ \\
\hline Set/07 & $0,078 \pm 2$ & $\pm 3,8 \mathrm{e}-4$ & -3 & -4 & -3 & -4 & $8 \mathrm{e}-4$ \\
\hline Out/07 & $0,035 \pm 5,6 \mathrm{e}-4$ & $0,029 \pm 6,1 \mathrm{e}-4$ & $0,029 \pm 5$ & $0,029 \pm 4,1 \mathrm{e}-4$ & $0,027 \pm 2,6 \mathrm{e}-4$ & $0,026 \pm$ & $0,037 \pm 9,1 \mathrm{e}-4$ \\
\hline No & $0,041 \pm 3$ & 0,0 & -4 & -4 & -3 & -4 & $9 \pm 4,8 \mathrm{e}-4$ \\
\hline Dez/07 & $0,073 \pm 1$ & $0,036 \pm 5$ & $0,033 \pm 9$ & $0,034 \pm$ & $0,034 \pm 9,7 \mathrm{e}-4$ & $0,039 \pm$ & $0,059 \pm 1,0 \mathrm{e}-3$ \\
\hline & 0,0 & & & & & & \\
\hline Fer & $0,047 \pm 6,4 \mathrm{e}-4$ & $0,042 \pm 8,5 \mathrm{e}-4$ & $0,041 \pm 7$ & $0,033 \pm$ & $0,032 \pm 9,6 \mathrm{e}-4$ & $0,035 \pm$ & $0,034 \pm 3,9 \mathrm{e}-4$ \\
\hline & $0,047 \pm 1$ & & & & $28 \pm 9$ & & $8 \mathrm{e}-4$ \\
\hline Ab & $0,032 \pm$ & $0,037 \pm 1$ & $0,032 \pm 4$ & $0,036 \pm$ & $0,036 \pm 8,2$ & $0,029 \pm$ & $0,028 \pm 7,2 \mathrm{e}-4$ \\
\hline Ma & $0,027 \pm 1$ & $0,021 \pm 8$ & \pm 1 & $0,017 \pm$ & $0,017 \pm 1$ & $6 \pm$ & $9 e-4$ \\
\hline Jur & $0,038 \pm 2$ & $0,019 \pm 1$ & $16 \pm 1$ & $0,018 \pm$ & $0,015 \pm 9$ & 0, & $0,017 \pm 1,2 \mathrm{e}-3$ \\
\hline Jul/08 & $0,030 \pm 1$ & $0,025 \pm 1$ & $17 \pm 1$ & $0,023 \pm$ & $0,019 \pm 1$ & $0,020 \pm$ & $0,016 \pm 2,6 \mathrm{e}-4$ \\
\hline Ago/08 & $0,046 \pm$ & $0,047 \pm 9$ & $43 \pm 1$ & $0,037 \pm$ & $0,044 \pm 6$ & $0,036 \pm$ & $0,041 \pm 4,4 \mathrm{e}-4$ \\
\hline Set/08 & $0,048 \pm 2$ & $0,040 \pm 7,7 \mathrm{e}-4$ & $0,045 \pm 5$ & $0,036 \pm 1,1 \mathrm{e}-3$ & $0,045 \pm 1,8 \mathrm{e}-3$ & $0,046 \pm$ & $0,035 \pm 2,0 \mathrm{e}-3$ \\
\hline Out/08 & $0,048 \pm 1,4 \mathrm{e}-3$ & $0,033 \pm 4$ & $0,048 \pm 1$ & $0,040 \pm 1,2 \mathrm{e}-3$ & $0,038 \pm 1$ & $0,044 \pm$ & $0,034 \pm 1,7 \mathrm{e}-3$ \\
\hline Nov/08 & $0,032 \pm 1,8 \mathrm{e}-3$ & $0,025 \pm 7,1 \mathrm{e}-4$ & $0,026 \pm 5,3 \mathrm{e}-4$ & $0,029 \pm 3,9 \mathrm{e}-4$ & $0,039 \pm 2,7 \mathrm{e}-3$ & $0,027 \pm 1,2 \mathrm{e}-4$ & $0,034 \pm 2,5 \mathrm{e}-4$ \\
\hline Dez/08 & $0,028 \pm 1,7 \mathrm{e}-4$ & $0,026 \pm 5,5 \mathrm{e}-4$ & $0,018 \pm 1,4 \mathrm{e}-3$ & $0,021 \pm 6,6 \mathrm{e}-4$ & $0,016 \pm 2,0 \mathrm{e}-4$ & $0,015 \pm 1,9 \mathrm{e}-4$ & $0,019 \pm 5,2 \mathrm{e}-4$ \\
\hline Jan/09 & $0,016 \pm 2,4 \mathrm{e}-4$ & $0,017 \pm 6,3 \mathrm{e}-4$ & $0,017 \pm 2,7 \mathrm{e}-4$ & $0,019 \pm 3,8 \mathrm{e}-4$ & $0,013 \pm 4,6 \mathrm{e}-4$ & $0,017 \pm 7,1 \mathrm{e}-4$ & $0,014 \pm 5,5 \mathrm{e}-4$ \\
\hline Fev/09 & $0,021 \pm 6,1 \mathrm{e}-4$ & $0,020 \pm 3,2 \mathrm{e}-4$ & $0,021 \pm 3,1 \mathrm{e}-4$ & $0,013 \pm 2,8 \mathrm{e}-4$ & $0,020 \pm 3,9 \mathrm{e}-4$ & $0,018 \pm 3,2 \mathrm{e}-4$ & $0,011 \pm 4,8 \mathrm{e}-4$ \\
\hline Mar/09 & $, 032 \pm 2,0 \mathrm{e}-4$ & $028 \pm 8,7 \mathrm{e}-4$ & $0,020 \pm 5,8 \mathrm{e}-4$ & $0,018 \pm 7$ & $0,021 \pm 4,1 \mathrm{e}-4$ & $0,021 \pm 1,2 \mathrm{e}-3$ & $0,014 \pm 3,1 \mathrm{e}-4$ \\
\hline
\end{tabular}

Os dados são médias de três leituras da amostra. 
Tabela 8 - Variação espaço-temporal de $\mathrm{Mn}\left(\mathrm{mg} . \mathrm{L}^{-1}\right)$ determinada no período de abril de 2007 a março de 2009 nos pontos de amostragem.

\begin{tabular}{|c|c|c|c|c|c|c|c|}
\hline Período & P1 & P2 & P3 & P4 & P5 & P6 & P7 \\
\hline Abr/07 & $037 \pm 3,7 \mathrm{e}-4$ & $, 048 \pm 2,3 \mathrm{e}-4$ & $063 \pm 3,7 \mathrm{e}-4$ & $0,031 \pm 1,7 \mathrm{e}-4$ & $0,023 \pm 2,4 \mathrm{e}-4$ & $0,005 \pm 2,3 \mathrm{e}-4$ & $0,009 \pm 3,1 \mathrm{e}-4$ \\
\hline Mai/07 & $0,041 \pm 5,9 \mathrm{e}-4$ & $0,041 \pm 6,7 \mathrm{e}-4$ & $0,048 \pm 4,2 \mathrm{e}-4$ & e-4 & $e-4$ & $09 \pm$ & $6,7 e-4$ \\
\hline Jun/07 & $0,038 \pm 2,8 \mathrm{e}-4$ & $0,037 \pm 3,7 \mathrm{e}-4$ & $0,045 \pm 4,4 \mathrm{e}-4$ & $0,035 \pm 5,2 \mathrm{e}-4$ & $0,021 \pm 4,6 \mathrm{e}-4$ & $0,008 \pm 5,1 \mathrm{e}-4$ & $011 \pm 4,7 \mathrm{e}-4$ \\
\hline Jul/07 & $0,035 \pm 5,7 \mathrm{e}-4$ & $0,042 \pm 1,1 \mathrm{e}-3$ & $0,048 \pm 7,6$ & $0,034 \pm 1,9 \mathrm{e}-4$ & $23 \pm 6$ & $0,007 \pm 2,9 \mathrm{e}-4$ & $0,009 \pm 6,6 \mathrm{e}-5$ \\
\hline Ago/07 & $034 \pm 3,2 \mathrm{e}-4$ & $0,036 \pm 8,7 \mathrm{e}-4$ & $0,045 \pm 4,4 \mathrm{e}-4$ & $0,056 \pm 1,2 \mathrm{e}-3$ & $19 \pm 3,2 \mathrm{e}-4$ & $0,008 \pm 2,7 \mathrm{e}-4$ & $0,010 \pm 1,5 \mathrm{e}-4$ \\
\hline Set/07 & $0,027 \pm 5,3 \mathrm{e}-4$ & $0,018 \pm 1,9 \mathrm{e}-4$ & $0,015 \pm 4,6$ & $0,007 \pm 8,9 \mathrm{e}-5$ & $03 \pm 1$, & $0,001 \pm 4,4 \mathrm{e}-4$ & $0,001 \pm 6,0 \mathrm{e}-5$ \\
\hline Out/07 & $78 \pm 8,3 e-4$ & $38 \pm 3,4 \mathrm{e}-4$ & $0,020 \pm 1,5$ & $43 \pm 1,5 \mathrm{e}-3$ & $27 \pm 6,7$ & $05 \pm 1,9 e-4$ & $0,004 \pm 1,4 \mathrm{e}-4$ \\
\hline Nov/07 & $0,055 \pm 2,4 \mathrm{e}-3$ & $0,031 \pm 9,4 \mathrm{e}-4$ & $0,031 \pm 1,0 \mathrm{e}-3$ & $0,035 \pm 1,1 \mathrm{e}-4$ & $0,019 \pm 1,7 \mathrm{e}-4$ & $0,006 \pm 1,7 \mathrm{e}-4$ & $0,008 \pm 2,3 \mathrm{e}-4$ \\
\hline Dez/07 & $37 \pm 5,2 \mathrm{e}-4$ & $0,026 \pm 2,6 \mathrm{e}-4$ & $0,022 \pm 6,5$ & $0,013 \pm$ & $7 \pm 4,5$ & $\pm 5,6 \mathrm{e}-4$ & $0,004 \pm 3,5 \mathrm{e}-4$ \\
\hline Jan/08 & $0,165 \pm 2,5 \mathrm{e}-3$ & $0,035 \pm 2,1 \mathrm{e}-3$ & $0,024 \pm 3,3 \mathrm{e}-4$ & $0,026 \pm 7,6 \mathrm{e}-4$ & $0,013 \pm 2,9$ & $0,005 \pm 3,1 \mathrm{e}-4$ & $0,005 \pm 2,3 \mathrm{e}-4$ \\
\hline Fev/08 & $\pm 1,4 \mathrm{e}-3$ & $\pm 1,2 \mathrm{e}-3$ & $8 \pm 6$ & $0,009 \pm 5,8 \mathrm{e}-4$ & $1 \pm 1,2$ & $\pm 3,9 \mathrm{e}-4$ & $4 \pm 7,8 \mathrm{e}-4$ \\
\hline Mar/08 & $0,049 \pm 2,1 \mathrm{e}-3$ & $0,045 \pm 1,6 \mathrm{e}-3$ & $0,048 \pm 1,3$ & $0,029 \pm 5,6 \mathrm{e}-4$ & $15 \pm 8,8$ & $0,005 \pm 2,5 \mathrm{e}-4$ & $0,006 \pm 3,5 \mathrm{e}-4$ \\
\hline Abr/08 & $43 \pm 1,6 e-3$ & $\pm 3,5 \mathrm{e}-5$ & $0,067 \pm 3,0 \mathrm{e}-4$ & $0,029 \pm 1,6 \mathrm{e}-3$ & $13 \pm 8,2 \mathrm{e}-4$ & $\pm 1,4 \mathrm{e}-4$ & $0,004 \pm 2,8 \mathrm{e}-4$ \\
\hline Mai/08 & $0,032 \pm 9,4 \mathrm{e}-4$ & $0,035 \pm 4,3 \mathrm{e}-4$ & $0,051 \pm 1,2 \mathrm{e}-3$ & $0,028 \pm 3,0 \mathrm{e}-4$ & $15 \pm 1,5$ & $0,005 \pm 4,5 \mathrm{e}-4$ & $0,007 \pm 1,3 \mathrm{e}-4$ \\
\hline Jun/08 & $0,030 \pm 6,8 \mathrm{e}-4$ & $0,029 \pm 3,2 \mathrm{e}-4$ & $\pm \pm 6,5 \mathrm{e}-4$ & $0,029 \pm 6,5 \mathrm{e}-4$ & $0,012 \pm 2,9 \mathrm{e}-4$ & $0,003 \pm 2,8 \mathrm{e}-4$ & $0,005 \pm 2,8 \mathrm{e}-4$ \\
\hline Jul/08 & $0,030 \pm 2,6 \mathrm{e}-4$ & $0,033 \pm 6,8 \mathrm{e}-4$ & $0,039 \pm 8,4 \mathrm{e}-4$ & $0,026 \pm 4,8 \mathrm{e}-4$ & $12 \pm 4,2 \mathrm{e}-4$ & $0,005 \pm 2,5 \mathrm{e}-4$ & $0,006 \pm 4,9 \mathrm{e}-4$ \\
\hline Ago/08 & $0,039 \pm 5,2 \mathrm{e}-4$ & $0,035 \pm 3,1 \mathrm{e}-4$ & $0,046 \pm 3,2 \mathrm{e}-4$ & $0,042 \pm 8,4 \mathrm{e}-4$ & $0,021 \pm 8,1 \mathrm{e}-4$ & $0,008 \pm 3,2 \mathrm{e}-4$ & $0,010 \pm 4,7 \mathrm{e}-4$ \\
\hline Set/08 & $0,034 \pm 3,3 \mathrm{e}-4$ & $0,019 \pm 1,5 \mathrm{e}-4$ & $0,015 \pm 1,2 \mathrm{e}-4$ & $0,010 \pm 2,7 \mathrm{e}-4$ & $0,007 \pm 2,7 \mathrm{e}-4$ & $0,004 \pm 1,8 \mathrm{e}-4$ & \\
\hline Out/08 & $0,014 \pm 9,1 \mathrm{e}-4$ & $0,001 \pm 2,0 \mathrm{e}-4$ & $0,002 \pm 2,0 \mathrm{e}-4$ & $0,002 \pm 1,7 \mathrm{e}-4$ & 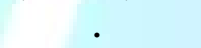 & . & . \\
\hline Nov/08 & $0,033 \pm 3,8 \mathrm{e}-4$ & $0,010 \pm 4,6 \mathrm{e}-4$ & $0,004 \pm 2,3 \mathrm{e}-4$ & $0,004 \pm 4,4 \mathrm{e}-4$ & $14 \pm 2,3 \mathrm{e}-4$ & $0,002 \pm 2,0 \mathrm{e}-4$ & $0,003 \pm 6,0 \mathrm{e}-4$ \\
\hline Dez/08 & $0,075 \pm 2,3 \mathrm{e}-3$ & $0,114 \pm 1,2 \mathrm{e}-3$ & $0,119 \pm 3,7 \mathrm{e}-3$ & $0,041 \pm 8,9 \mathrm{e}-4$ & $0,012 \pm 1,7 \mathrm{e}-3$ & $0,005 \pm 7,2 \mathrm{e}-4$ & $0,006 \pm 6,1 \mathrm{e}-4$ \\
\hline Jan/09 & $0,077 \pm 4,5 \mathrm{e}-4$ & $0,068 \pm 3,7 \mathrm{e}-4$ & $0,051 \pm 5,6 \mathrm{e}-4$ & $0,078 \pm 8,0 \mathrm{e}-4$ & $0,042 \pm 4,9 \mathrm{e}-4$ & $0,022 \pm 6,7 \mathrm{e}-4$ & $0,018 \pm 7,1 \mathrm{e}-4$ \\
\hline Fev/09 & $0,065 \pm 6,2 \mathrm{e}-4$ & $0,090 \pm 7,7 \mathrm{e}-4$ & $0,075 \pm 5,4 \mathrm{e}-4$ & $0,077 \pm 6,5 \mathrm{e}-4$ & $0,456 \pm 8,3 \mathrm{e}-3$ & $0,022 \pm 5,5 \mathrm{e}-4$ & $0,020 \pm 6,5 \mathrm{e}-4$ \\
\hline Mar/09 & $0,067 \pm 5,1 \mathrm{e}-4$ & $0,129 \pm 6,7 \mathrm{e}-4$ & $0,127 \pm 5,2 \mathrm{e}-4$ & $0,066 \pm 6,1 \mathrm{e}-4$ & $0,049 \pm 5,9 \mathrm{e}-4$ & $0,020 \pm 7,1 \mathrm{e}-4$ & $0,021 \pm 4,3 \mathrm{e}-4$ \\
\hline
\end{tabular}

Legenda: $(\cdot)$ = não detectado. Os dados são médias de três leituras da amostra.

Tabela 9 - Variação espaço-temporal de Ca $\left(\mathrm{mg} \cdot \mathrm{L}^{-1}\right)$ determinada no período de abril de 2007 a março de 2009 nos pontos de amostragem.

\begin{tabular}{|c|c|c|c|c|c|c|c|}
\hline Período & P1 & P2 & P3 & P4 & P5 & P6 & P7 \\
\hline Abr/07 & $1,58 \pm 2,1 \mathrm{e}-2$ & $2,33 \pm 3,1 \mathrm{e}-2$ & $2,63 \pm 3,2 \mathrm{e}-2$ & $3,10 \pm 4,8 \mathrm{e}-2$ & $3,60 \pm 5,8 \mathrm{e}-2$ & $4,11 \pm 3,7 e-2$ & $3,89 \pm 2,9 \mathrm{e}-2$ \\
\hline Mai/07 & $1,03 \pm 5,2 \mathrm{e}-3$ & $1,73 \pm 2,2 \mathrm{e}-2$ & $2,24 \pm 3,4 \mathrm{e}-2$ & $2,60 \pm 2,2 \mathrm{e}-2$ & $3,18 \pm 1,9 \mathrm{e}-2$ & $3,95 \pm 1,7 \mathrm{e}-2$ & $3,61 \pm 1,2 \mathrm{e}-2$ \\
\hline Jun/07 & $1,19 \pm 2,7 \mathrm{e}-3$ & $1,69 \pm 2,3 \mathrm{e}-2$ & $2,14 \pm 1,3 \mathrm{e}-2$ & $2,51 \pm 1,7 \mathrm{e}-2$ & $2,88 \pm 2,2 \mathrm{e}-2$ & $3,48 \pm 2,4 \mathrm{e}-2$ & $3,34 \pm 3,0 \mathrm{e}-2$ \\
\hline Jul/07 & $1,11 \pm 4,2 \mathrm{e}-3$ & $1,77 \pm 1,1 \mathrm{e}-2$ & $2,36 \pm 1,4 \mathrm{e}-2$ & $2,57 \pm 1,1 \mathrm{e}-2$ & $2,99 \pm 2,9 \mathrm{e}-2$ & $3,38 \pm 2,6 e-2$ & $3,36 \pm 1,8 \mathrm{e}-2$ \\
\hline Ago/07 & $1,29 \pm 3,7 \mathrm{e}-3$ & $1,76 \pm 1,4 \mathrm{e}-2$ & $2,27 \pm 2,0 \mathrm{e}-2$ & $2,74 \pm 3,7 \mathrm{e}-3$ & $3,15 \pm 3,7 e-2$ & $4,00 \pm 1,8 \mathrm{e}-2$ & $3,64 \pm 2,8 \mathrm{e}-2$ \\
\hline Set/07 & $1,53 \pm 1,6 \mathrm{e}-2$ & $1,67 \pm 2,1 \mathrm{e}-2$ & $2,13 \pm 3,4 \mathrm{e}-3$ & $2,52 \pm 2,8 \mathrm{e}-2$ & $3,11 \pm 1,1 \mathrm{e}-2$ & $3,85 \pm 4,2 \mathrm{e}-2$ & $3,51 \pm 8,5 \mathrm{e}-2$ \\
\hline Out/07 & $1,63 \pm 2,5 \mathrm{e}-2$ & $1,97 \pm 2,1 \mathrm{e}-2$ & $2,49 \pm 2,1 \mathrm{e}-2$ & $2,70 \pm 2,5 \mathrm{e}-2$ & $3,05 \pm 2,1 \mathrm{e}-2$ & $4,01 \pm 1,1 \mathrm{e}-2$ & $3,55 \pm 2,4 \mathrm{e}-2$ \\
\hline Nov/07 & $1,35 \pm 2,0 \mathrm{e}-3$ & $1,95 \pm 8,7 \mathrm{e}-3$ & $2,46 \pm 1,8 \mathrm{e}-2$ & $2,93 \pm 2,7 \mathrm{e}-2$ & $3,29 \pm 5,4 \mathrm{e}-2$ & $3,82 \pm 2,5 e-2$ & $3,69 \pm 1,3 e-2$ \\
\hline Dez/07 & $1,76 \pm 1,3 e-2$ & $1,92 \pm 9,1 \mathrm{e}-3$ & $2,38 \pm 2,8 \mathrm{e}-2$ & $2,53 \pm 2,5 \mathrm{e}-2$ & $2,93 \pm 2,0 \mathrm{e}-2$ & $3,48 \pm 5,4 e-2$ & $3,59 \pm 1,4 \mathrm{e}-2$ \\
\hline $\mathrm{Jan} / 08$ & $1,46 \pm 1,8 \mathrm{e}-2$ & $2,31 \pm 1,2 \mathrm{e}-2$ & $2,79 \pm 1,3 e-2$ & $3,29 \pm 5,8 \mathrm{e}-2$ & $3,48 \pm 1,3 e-2$ & $4,53 \pm 3,0 \mathrm{e}-2$ & $4,17 \pm 4,2 \mathrm{e}-2$ \\
\hline Fev/08 & $1,27 \pm 1,7 \mathrm{e}-2$ & $2,00 \pm 3,2 \mathrm{e}-2$ & $2,29 \pm 5,2 \mathrm{e}-2$ & $3,21 \pm 5,6 \mathrm{e}-2$ & $3,53 \pm 5,5 e-3$ & $3,23 \pm 2,1 \mathrm{e}-2$ & $3,42 \pm 4,1 \mathrm{e}-2$ \\
\hline Mar/08 & $2,29 \pm 2,1 e-2$ & $1,34 \pm 1,2 \mathrm{e}-2$ & $2,75 \pm 1,4 \mathrm{e}-2$ & $3,21 \pm 4,2 \mathrm{e}-2$ & $3,46 \pm 4,0 \mathrm{e}-2$ & $4,19 \pm 2,5 e-2$ & $3,88 \pm 4,4 \mathrm{e}-2$ \\
\hline Abr $/ 08$ & $1,14 \pm 1,2 \mathrm{e}-2$ & $1,98 \pm 2,1 \mathrm{e}-2$ & $2,54 \pm 7,9 \mathrm{e}-3$ & $3,00 \pm 3,3 \mathrm{e}-2$ & $3,14 \pm 3,0 \mathrm{e}-2$ & $3,91 \pm 4,6 \mathrm{e}-2$ & $3,62 \pm 3,3 \mathrm{e}-2$ \\
\hline Mai/08 & $1,15 \pm 1,5 \mathrm{e}-2$ & $1,76 \pm 1,3 \mathrm{e}-2$ & $2,16 \pm 2,5 \mathrm{e}-2$ & $2,60 \pm 3,8 \mathrm{e}-3$ & $2,93 \pm 4,3 e-2$ & $3,60 \pm 3,8 \mathrm{e}-2$ & $3,41 \pm 6,5 \mathrm{e}-2$ \\
\hline Jun/08 & $1,15 \pm 1,4 \mathrm{e}-2$ & $1,68 \pm 1,6 \mathrm{e}-2$ & $2,13 \pm 1,9 \mathrm{e}-2$ & $2,42 \pm 8,6 \mathrm{e}-3$ & $2,76 \pm 4,7 \mathrm{e}-2$ & $3,71 \pm 2,6 e-2$ & $3,61 \pm 4,5 \mathrm{e}-2$ \\
\hline Jul/08 & $0,99 \pm 1,1 \mathrm{e}-2$ & $1,55 \pm 2,4 \mathrm{e}-2$ & $1,85 \pm 1,3 \mathrm{e}-2$ & $2,20 \pm 1,7 \mathrm{e}-2$ & $2,58 \pm 4,1 \mathrm{e}-2$ & $3,51 \pm 4,4 \mathrm{e}-2$ & $3,19 \pm 6,2 \mathrm{e}-2$ \\
\hline Ago/08 & $1,25 \pm 1,4 \mathrm{e}-2$ & $1,83 \pm 2,7 \mathrm{e}-2$ & $2,17 \pm 9,9 \mathrm{e}-3$ & $2,61 \pm 1,5 \mathrm{e}-2$ & $3,09 \pm 3,0 \mathrm{e}-2$ & $3,95 \pm 3,3 e-2$ & $3,49 \pm 3,8 \mathrm{e}-2$ \\
\hline Set $/ 08$ & $1,21 \pm 1,9 \mathrm{e}-2$ & $1,68 \pm 4,1 \mathrm{e}-2$ & $1,83 \pm 8,5 \mathrm{e}-3$ & $2,06 \pm 9,6 \mathrm{e}-3$ & $2,41 \pm 2,6 \mathrm{e}-2$ & $3,38 \pm 7,5 \mathrm{e}-2$ & $2,95 \pm 1,5 \mathrm{e}-2$ \\
\hline Out $/ 08$ & $1,36 \pm 1,7 \mathrm{e}-2$ & $1,71 \pm 2,9 \mathrm{e}-2$ & $2,31 \pm 2,6 \mathrm{e}-2$ & $2,57 \pm 9,6 \mathrm{e}-3$ & $2,90 \pm 1,4 \mathrm{e}-2$ & $3,48 \pm 2,3 e-2$ & $3,38 \pm 5,8 \mathrm{e}-2$ \\
\hline Nov/08 & $1,28 \pm 3,0 \mathrm{e}-2$ & $1,79 \pm 1,9 \mathrm{e}-2$ & $2,23 \pm 3,2 \mathrm{e}-2$ & $2,37 \pm 6,6 \mathrm{e}-3$ & $2,78 \pm 4,4 \mathrm{e}-2$ & $3,47 \pm 3,1 \mathrm{e}-2$ & $3,21 \pm 2,2 \mathrm{e}-2$ \\
\hline Dez/08 & $1,30 \pm 2,2 \mathrm{e}-2$ & $2,18 \pm 3,8 \mathrm{e}-2$ & $3,04 \pm 4,0 \mathrm{e}-2$ & $2,90 \pm 9,8 \mathrm{e}-3$ & $2,83 \pm 3,3 \mathrm{e}-2$ & $4,34 \pm 6,1 \mathrm{e}-2$ & $3,59 \pm 5,2 \mathrm{e}-2$ \\
\hline Jan/09 & $1,63 \pm 1,8 \mathrm{e}-2$ & $2,42 \pm 2,4 \mathrm{e}-2$ & $3,17 \pm 2,9 \mathrm{e}-2$ & $3,77 \pm 1,5 \mathrm{e}-2$ & $4,21 \pm 2,7 \mathrm{e}-2$ & $11,99 \pm 3,0 \mathrm{e}-2$ & $8,57 \pm 3,2 \mathrm{e}-2$ \\
\hline Fev/09 & $1,77 \pm 3,8 \mathrm{e}-2$ & $2,57 \pm 4,5 \mathrm{e}-2$ & $3,19 \pm 3,7 \mathrm{e}-2$ & $3,80 \pm 2,7 \mathrm{e}-2$ & $4,87 \pm 4,9 \mathrm{e}-2$ & $7,84 \pm 6,6 e-2$ & $5,99 \pm 8,9 \mathrm{e}-2$ \\
\hline Mar/09 & $1,73 \pm 2,1 \mathrm{e}-2$ & $2,58 \pm 1,9 \mathrm{e}-2$ & $3,16 \pm 2,2 \mathrm{e}-2$ & $3,71 \pm 2,6 \mathrm{e}-2$ & $4,44 \pm 3,3 e-2$ & $5,83 \pm 2,1 \mathrm{e}-2$ & $5,27 \pm 3,7 \mathrm{e}-2$ \\
\hline
\end{tabular}

Os dados são médias de três leituras da amostra. 
Tabela 10 - Variação espaço-temporal de $\mathrm{Mg}\left(\mathrm{mg} \cdot \mathrm{L}^{-1}\right)$ determinada no período de abril de 2007 a março de 2009 nos pontos de amostragem.

\begin{tabular}{|c|c|c|c|c|c|c|c|}
\hline Período & P1 & P2 & P3 & P4 & P5 & P6 & P7 \\
\hline Abr/07 & $0,89 \pm 7,1 \mathrm{e}-3$ & $0,97 \pm 6,4 \mathrm{e}-3$ & $1,10 \pm 7,5 \mathrm{e}-3$ & $1,24 \pm 4,4 \mathrm{e}-3$ & $1,56 \pm 6,6 \mathrm{e}-3$ & $2,91 \pm 4,5 \mathrm{e}-3$ & $2,31 \pm 6,7 \mathrm{e}-3$ \\
\hline Mai/07 & $0,69 \pm 5,3 \mathrm{e}-3$ & $0,75 \pm 4,8 \mathrm{e}-3$ & $0,93 \pm 5,5 \mathrm{e}-3$ & $1,04 \pm 7,9 \mathrm{e}-3$ & $1,31 \pm 8,4 \mathrm{e}-3$ & $2,89 \pm 7,2 \mathrm{e}-3$ & $2,13 \pm 8,2 \mathrm{e}-3$ \\
\hline Jun/07 & $0,78 \pm 5,1 \mathrm{e}-3$ & $0,79 \pm 4,2 \mathrm{e}-3$ & $0,88 \pm 6,7 \mathrm{e}-3$ & $0,99 \pm 8,3 \mathrm{e}-3$ & $1,22 \pm, 4 \mathrm{e}-3$ & $2,60 \pm 6,4 \mathrm{e}-3$ & $2,02 \pm 4,7 \mathrm{e}-3$ \\
\hline Jul/07 & $0,77 \pm 3,4 \mathrm{e}-3$ & $0,84 \pm 7,3 \mathrm{e}-3$ & $1,01 \pm 1,3 \mathrm{e}-2$ & $1,10 \pm 7,4 \mathrm{e}-3$ & $1,34 \pm 1,4 \mathrm{e}-2$ & $2,45 \pm 1,7 \mathrm{e}-2$ & $2,16 \pm 1,8 \mathrm{e}-2$ \\
\hline Ago/07 & $0,84 \pm 6,1 \mathrm{e}-3$ & $0,84 \pm 6,8 \mathrm{e}-3$ & $0,95 \pm 3,5 \mathrm{e}-3$ & $1,12 \pm 1,3 \mathrm{e}-2$ & $1,39 \pm 3,5 \mathrm{e}-2$ & $2,98 \pm 2,9 \mathrm{e}-2$ & $2,31 \pm 4,5 \mathrm{e}-3$ \\
\hline Set $/ 07$ & $0,94 \pm 1,8 \mathrm{e}-2$ & $0,80 \pm 6,8 \mathrm{e}-3$ & $0,92 \pm 1,2 \mathrm{e}-2$ & $1,01 \pm 1,2 \mathrm{e}-2$ & $1,35 \pm 6,8 \mathrm{e}-3$ & $2,65 \pm 2,4 \mathrm{e}-2$ & $2,01 \pm 1,0 \mathrm{e}-2$ \\
\hline Out/07 & $1,07 \pm 6,3 \mathrm{e}-3$ & $0,95 \pm 3,1 \mathrm{e}-3$ & $1,08 \pm 1,1 \mathrm{e}-2$ & $1,18 \pm 8,9 \mathrm{e}-3$ & $1,36 \pm 5,3 \mathrm{e}-3$ & $3,03 \pm 1,8 \mathrm{e}-2$ & $2,21 \pm 1,9 \mathrm{e}-2$ \\
\hline Nov/07 & $0,85 \pm 8,1 \mathrm{e}-3$ & $0,86 \pm 9,5 \mathrm{e}-3$ & $1,00 \pm 3,3 e-3$ & $1,15 \pm 6,6 \mathrm{e}-3$ & $1,41 \pm 1,4 \mathrm{e}-2$ & $2,72 \pm 1,1 \mathrm{e}-2$ & $2,15 \pm 5,9 \mathrm{e}-3$ \\
\hline Dez/07 & $0,87 \pm 7,0 \mathrm{e}-3$ & $0,85 \pm 7,4 \mathrm{e}-3$ & $1,02 \pm 1,3 \mathrm{e}-2$ & $1,24 \pm 4,5 \mathrm{e}-3$ & $1,67 \pm 1,6 \mathrm{e}-2$ & $2,16 \pm 7,5 \mathrm{e}-3$ & $2,18 \pm 8,0 \mathrm{e}-3$ \\
\hline Jan/08 & $1,48 \pm 9,9 \mathrm{e}-3$ & $0,94 \pm 1,4 \mathrm{e}-2$ & $1,09 \pm 9,7 \mathrm{e}-3$ & $1,29 \pm 6,6 \mathrm{e}-3$ & $1,50 \pm 9,7 \mathrm{e}-3$ & $3,47 \pm 4,3 \mathrm{e}-2$ & $2,81 \pm 4,4 \mathrm{e}-2$ \\
\hline Fev/08 & $0,69 \pm 3,6 \mathrm{e}-3$ & $0,82 \pm 3,9 \mathrm{e}-3$ & $0,95 \pm 1,4 \mathrm{e}-2$ & $1,28 \pm 6,3 e-3$ & $1,48 \pm 2,1 \mathrm{e}-2$ & $1,96 \pm 1,2 \mathrm{e}-2$ & $1,93 \pm 4,9 \mathrm{e}-3$ \\
\hline Mar/08 & $0,94 \pm 5,6 \mathrm{e}-3$ & $0,82 \pm 5,3 \mathrm{e}-3$ & $1,06 \pm 7,9 \mathrm{e}-3$ & $1,25 \pm 1,4 \mathrm{e}-2$ & $1,46 \pm 1,8 \mathrm{e}-2$ & $3,17 \pm 2,5 \mathrm{e}-2$ & $2,52 \pm 1,6 \mathrm{e}-2$ \\
\hline Abr/08 & $0,75 \pm 3,9 \mathrm{e}-3$ & $0,88 \pm 3,2 \mathrm{e}-3$ & $1,03 \pm 2,7 \mathrm{e}-2$ & $1,26 \pm 9,5 \mathrm{e}-3$ & $1,36 \pm 1,3 \mathrm{e}-2$ & $2,84 \pm 2,0 \mathrm{e}-2$ & $2,23 \pm 2,0 \mathrm{e}-2$ \\
\hline Mai/08 & $0,67 \pm 4,5 \mathrm{e}-3$ & $0,75 \pm 8,5 \mathrm{e}-4$ & $0,86 \pm 1,2 \mathrm{e}-2$ & $1,03 \pm 3,8 \mathrm{e}-3$ & $1,21 \pm 4,3 \mathrm{e}-2$ & $2,66 \pm 3,8 \mathrm{e}-2$ & $2,12 \pm 1,2 \mathrm{e}-2$ \\
\hline Jun/08 & $0,74 \pm 5,0 \mathrm{e}-3$ & $0,74 \pm 6,7 \mathrm{e}-3$ & $0,86 \pm 5,7 \mathrm{e}-3$ & $0,96 \pm 1,0 \mathrm{e}-2$ & $1,13 \pm 3,4 \mathrm{e}-2$ & $2,68 \pm 1,8 \mathrm{e}-2$ & $2,21 \pm 1,0 \mathrm{e}-2$ \\
\hline Jul/08 & $0,81 \pm 4,4 \mathrm{e}-3$ & $0,71 \pm 6,2 \mathrm{e}-3$ & $0,79 \pm 2,6 \mathrm{e}-3$ & $0,89 \pm 2,0 \mathrm{e}-3$ & $1,08 \pm 4,9 \mathrm{e}-2$ & $2,60 \pm 1,2 \mathrm{e}-2$ & $1,96 \pm 1,6 \mathrm{e}-2$ \\
\hline Ago/08 & $0,80 \pm 6,1 \mathrm{e}-3$ & $0,82 \pm 4,3 e-3$ & $0,91 \pm 9,5 \mathrm{e}-3$ & $1,06 \pm 1,6 \mathrm{e}-2$ & $1,27 \pm 1,5 \mathrm{e}-2$ & $2,73 \pm 1,4 \mathrm{e}-2$ & $2,08 \pm 4,2 \mathrm{e}-2$ \\
\hline Set $/ 08$ & $0,75 \pm 4,5 \mathrm{e}-3$ & $0,76 \pm 1,9 \mathrm{e}-2$ & $0,83 \pm 7,4 \mathrm{e}-3$ & $0,90 \pm 1,2 \mathrm{e}-2$ & $1,05 \pm 1,6 \mathrm{e}-2$ & $2,60 \pm 2,6 e-2$ & $1,85 \pm 1,5 \mathrm{e}-2$ \\
\hline Out/08 & $0,86 \pm 3,4 \mathrm{e}-3$ & $0,78 \pm 1,7 \mathrm{e}-2$ & $0,90 \pm 8,4 \mathrm{e}-3$ & $1,01 \pm 1,3 \mathrm{e}-2$ & $1,20 \pm 1,6 \mathrm{e}-2$ & $2,12 \pm 1,8 \mathrm{e}-2$ & $1,79 \pm 2,9 \mathrm{e}-2$ \\
\hline Nov/08 & $0,78 \pm 7,5 \mathrm{e}-3$ & $0,80 \pm 1,5 \mathrm{e}-2$ & $0,92 \pm 1,3 \mathrm{e}-2$ & $0,98 \pm 4,7 \mathrm{e}-3$ & $1,21 \pm 1,0 \mathrm{e}-2$ & $2,33 \pm 2,2 \mathrm{e}-2$ & $1,83 \pm 2,3 e-2$ \\
\hline Dez/08 & $0,82 \pm 5,6 e-3$ & $0,97 \pm 1,0 \mathrm{e}-2$ & $1,29 \pm 2,9 \mathrm{e}-2$ & $1,30 \pm 8,9 \mathrm{e}-3$ & $1,38 \pm 2,7 \mathrm{e}-2$ & $3,29 \pm 1,1 \mathrm{e}-2$ & $2,34 \pm 2,2 \mathrm{e}-2$ \\
\hline Jan/09 & $0,99 \pm 6,2 \mathrm{e}-3$ & $1,02 \pm 1,7 \mathrm{e}-2$ & $1,28 \pm 2,3 \mathrm{e}-2$ & $1,57 \pm 1,8 \mathrm{e}-2$ & $1,89 \pm 1,3 \mathrm{e}-2$ & $12,58 \pm 6,5 \mathrm{e}-2$ & $7,80 \pm 1,8 \mathrm{e}-2$ \\
\hline Fev/09 & $1,02 \pm 4,1 \mathrm{e}-3$ & $1,11 \pm 2,1 \mathrm{e}-2$ & $1,30 \pm 3,0 \mathrm{e}-2$ & $1,57 \pm 2,8 \mathrm{e}-2$ & $1,98 \pm 2,4 \mathrm{e}-2$ & $7,99 \pm 2,4 \mathrm{e}-2$ & $5,88 \pm 3,1 \mathrm{e}-2$ \\
\hline Mar/09 & $1,07 \pm 3,8 \mathrm{e}-3$ & $1,13 \pm 9,7 \mathrm{e}-3$ & $1,31 \pm 8,7 \mathrm{e}-3$ & $1,56 \pm 1,2 \mathrm{e}-2$ & $2,06 \pm 2,1 \mathrm{e}-2$ & $5,60 \pm 1,7 \mathrm{e}-2$ & $4,13 \pm 1,5 \mathrm{e}-2$ \\
\hline
\end{tabular}

Os dados são médias de três leituras da amostra.

As médias dos períodos seco e chuvoso para os metais avaliados são apresentadas na Figura 5. Observa-se que com exceção do $\mathrm{Zn}$, as maiores médias foram observadas no período de maior pluviosidade, consequência provável do carreamento mais intenso de sedimentos para os corpos hídricos.

Para o período chuvoso, a Análise dos Componentes Principais (ACP) resumiu em seus dois primeiros componentes $81,96 \%$ da variabilidade dos dados de acréscimos de $\mathrm{Fe}, \mathrm{Mn}, \mathrm{Zn}, \mathrm{Al}, \mathrm{Ca}$ e $\mathrm{Mg}$, nas águas superficiais coletadas nos pontos de amostragens. O componente 1 explicou 59,02\% da variância total encontrada, enquanto que o componente 2 explicou 22,94\%. Os acréscimos de $\mathrm{Mg}, \mathrm{Al}$, e Ca apresentaram correlação positiva com o componente 1, enquanto que os acréscimos de $\mathrm{Mn}$ e $\mathrm{Zn}$ apresentaram correlação negativa. No componente 2, os acréscimos de Fe apresentaram correlação positiva (Tabela 11).

Após a ordenação pela análise de componentes principais, os pontos de amostragem no período chuvoso apresentaram uma distribuição desde a nascente da microbacia do rio Cabeça até a foz apresentando características discriminantes dos pontos P6 e P7, situados no rio Passa Cinco, como observado na Figura 6. Analisando $\mathrm{Ca}, \mathrm{Mg}$ e $\mathrm{Al}$ verifica-se que os pontos P6 e P7 apresentaram os maiores valores, enquanto que para o $\mathrm{Mn}$ e $\mathrm{Zn}$ os maiores valores foram obtidos nos pontos $\mathrm{P} 1, \mathrm{P} 2$ e $\mathrm{P} 3$. 

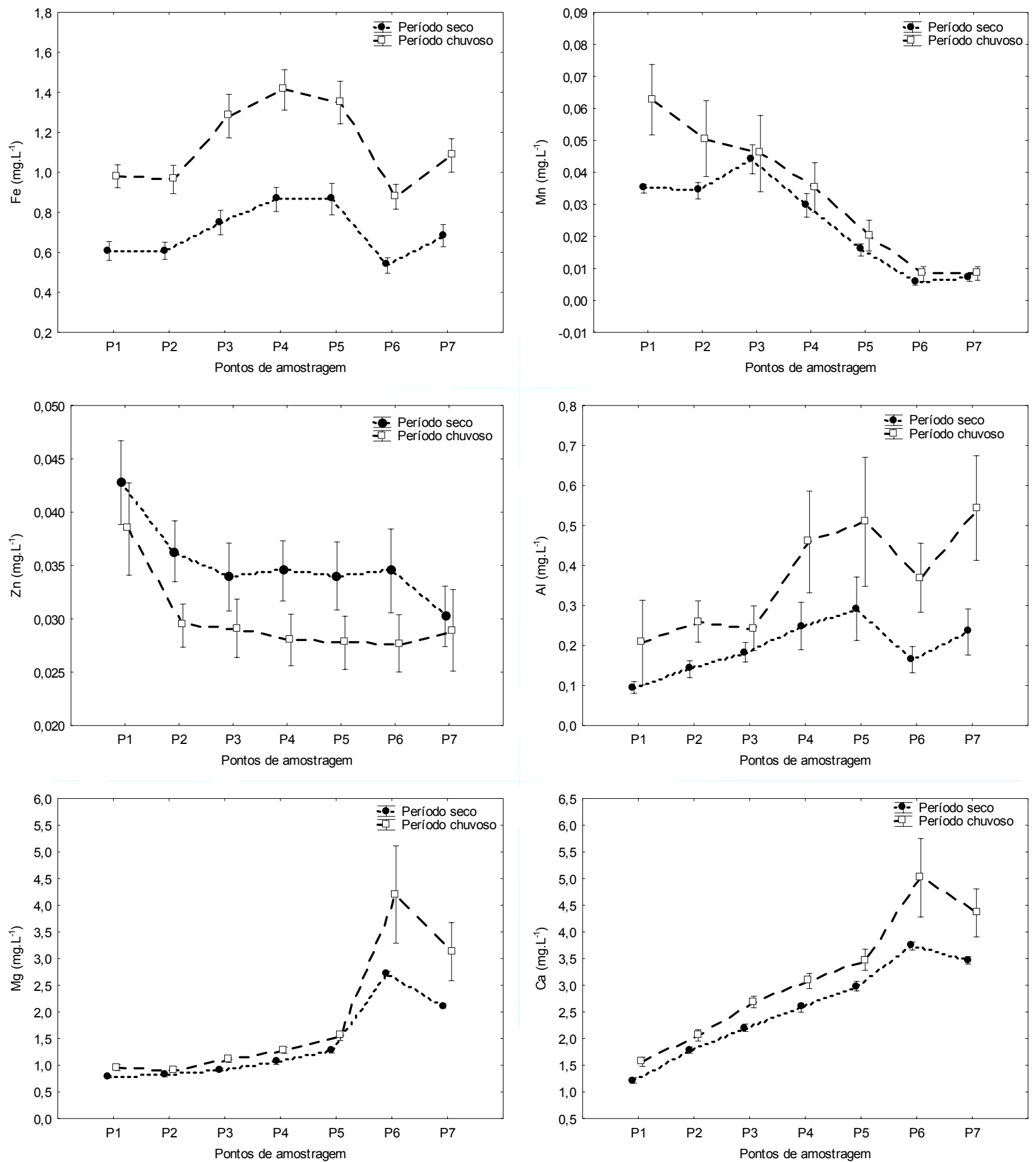

Figura 5. Médias das concentrações de metais em amostras de água do rio Cabeça (P1 a P5) e rio Passa Cinco (P6 e P7) nos períodos seco (abril a setembro) e chuvoso (outubro a março), de abril de 2007 a março de 2009. 
Tabela 11 - Correlação dos acréscimos de Fe, Mn, Zn, Al, Ca e Mg nas águas superficiais dos pontos amostrados no período chuvoso.

\begin{tabular}{ccc}
\hline Variáveis & Componente 1 & Componente 2 \\
\hline $\mathbf{F e}$ & $-0,098$ & $\mathbf{0 , 9 4 9}$ \\
$\mathbf{M g}$ & $\mathbf{0 , 8 5 2}$ & $-0,432$ \\
$\mathbf{M n}$ & $\mathbf{- 0 , 9 5 5}$ & $-0,085$ \\
$\mathbf{A l}$ & $\mathbf{0 , 8 4 9}$ & 0,495 \\
$\mathbf{Z n}$ & $\mathbf{- 0 , 7 4 3}$ & $-0,110$ \\
$\mathbf{C a}$ & $\mathbf{0 , 7 8 7}$ & $-0,154$ \\
\hline Variação explicada & $59,02 \%$ & $22,94 \%$ \\
\hline
\end{tabular}

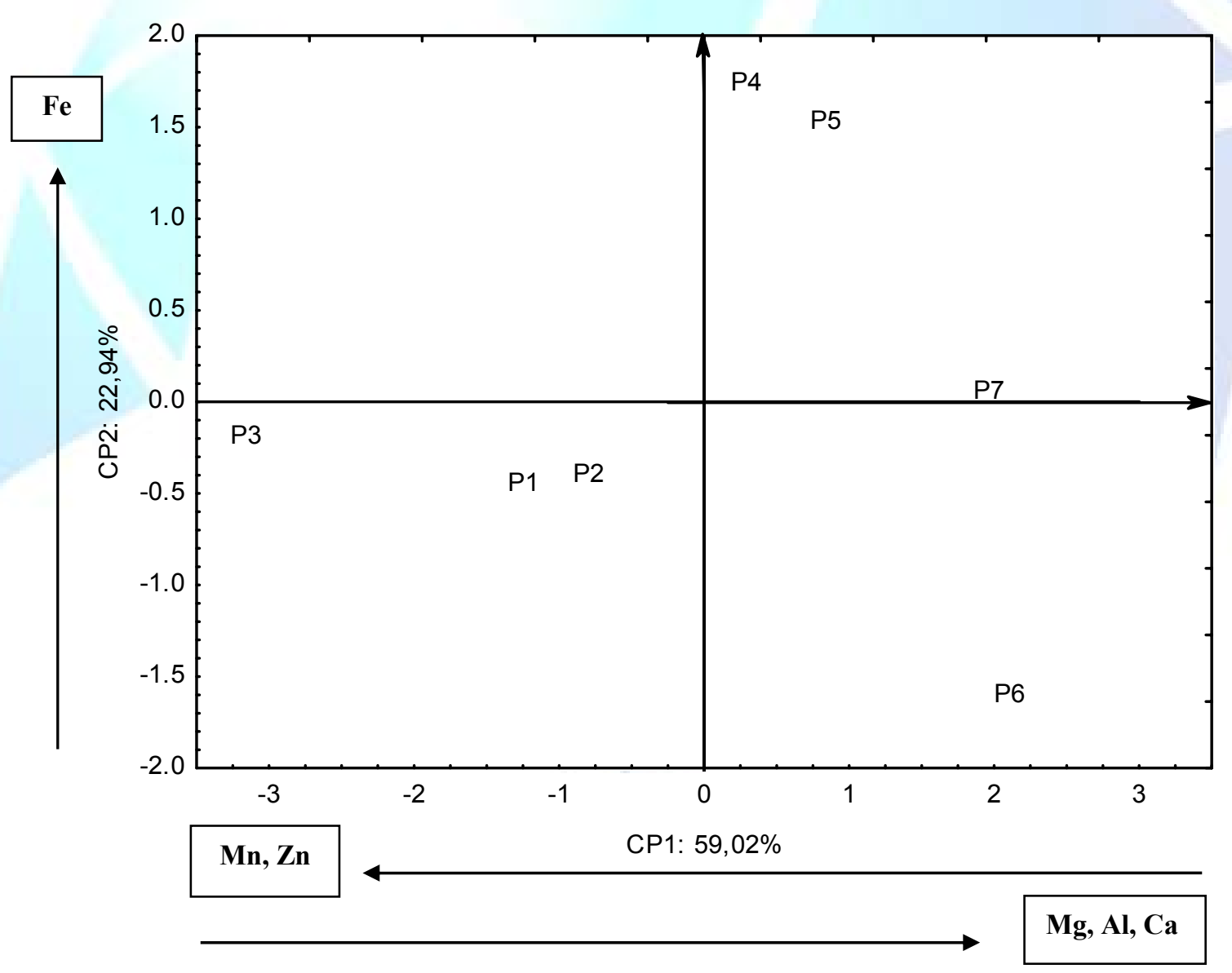

Figura 6. Ordenação pela ACP dos metais nas águas superficiais dos pontos amostrados no período chuvoso. 
Ainda segundo os resultados citados na Figura 5 houve acumulação de $\mathrm{Fe}$ no período chuvoso ao longo da microbacia do rio Cabeça. As características pedológicas é o principal fator determinante deste acúmulo, pois nesta microbacia há predomínio de solos podzólico avermelhado amarelo seguido por latossolo vermelho escuro, solos estes ricos em Fe (Figura 2). Tauk-Tornisielo et al. (2008) destacaram a importância dos solos na determinação da concentração de Fe em estudo realizado no rio Corumbataí. Esses autores encontraram concentrações próximas a $20 \mathrm{mg}$. $\mathrm{L}^{-1}$ valores estes muito superiores ao limite máximo de Fe permitido de $0,3 \mathrm{mg}$. $\mathrm{L}^{-1}$ fixado pela Resolução CONAMA 357/2005 (BRASIL, 2005), para corpos hídricos de classe 2. Foram observados valores de Fe acima do padrão em diversos pesque-pague ao longo da bacia do rio Corumbataí sendo que os valores encontrados para este parâmetro variaram de $0,01 \mathrm{mg} \cdot \mathrm{L}^{-1}$ a 9,5 mg.L ${ }^{-1}$ (TAUK-TORNISIELO, 2010). Os valores encontrados neste estudo para o Fe nos pontos P1 a P7 foram superiores ao limite máximo estabelecido pela legislação, citada anteriormente, tanto no período chuvoso quanto no período seco (Tabela 5).

$\mathrm{Da}$ mesma forma que o Fe, o Al tem grande ocorrência natural nos tipos de solos da microbacia estudada sendo carreado em maiores quantidades para os corpos superficiais com as chuvas. A Resolução CONAMA 357/2005 (BRASIL, 2005) fixou o limite de 0,2 mg.L $\mathrm{L}^{-1}$ para os rios de classe 2 . No período chuvoso $54,8 \%$ dos valores encontrados para este parâmetro estiveram acima deste limite máximo enquanto que no período seco $19,0 \%$ (Tabela 12).

Para o período seco, a Análise dos Componentes Principais (ACP) resumiu em seus dois primeiros componentes $92,62 \%$ da variabilidade dos dados de acréscimos de $\mathrm{Fe}, \mathrm{Mn}, \mathrm{Zn}, \mathrm{Al}, \mathrm{Ca}$ e $\mathrm{Mg}$ nas águas superficiais coletadas nos pontos de amostragem. O componente 1 explicou 60,17\% da variância total encontrada, enquanto que o componente 2 explicou 32,45\%. Os acréscimos de $\mathrm{Mg}, \mathrm{Al}$ e Ca apresentaram correlação positiva com o componente 1, enquanto que os acréscimos de Mn e Zn apresentaram correlação negativa. Já os acréscimos de Fe apresentaram correlação negativa com o componente 2 (Tabela 6).

Tabela 12 - Correlação dos acréscimos de $\mathrm{Fe}, \mathrm{Mg}, \mathrm{Mn}, \mathrm{Al}, \mathrm{Zn}$ e $\mathrm{Ca}$ nas águas superficiais dos pontos amostrados no período seco.

\begin{tabular}{c|cc}
\hline Variáveis & Componente 1 & Componente 2 \\
\hline $\mathbf{F e}$ & 0,175 & $\mathbf{- 0 , 9 6 6}$ \\
$\mathbf{M g}$ & $\mathbf{0 , 8 1 7}$ & 0,557 \\
$\mathbf{M n}$ & $\mathbf{- 0 , 8 6 0}$ & 0,363 \\
$\mathbf{A l}$ & $\mathbf{0 , 7 0 4}$ & $-0,694$ \\
$\mathbf{Z n}$ & $\mathbf{- 0 , 8 4 2}$ & 0,272 \\
$\mathbf{C a}$ & $\mathbf{0 , 9 8 3}$ & 0,122 \\
\hline Variação explicada & $60,17 \%$ & $32,45 \%$ \\
\hline
\end{tabular}

Após a ordenação pela análise de componentes principais, que explicou $92,6 \%$ da variabilidade dos dados, os pontos de amostragem no período seco apresentaram uma distribuição desde a nascente da microbacia do rio Cabeça até a foz, onde apresentaram características discriminantes dos pontos P6 e P7, ambos no rio Passa Cinco (Figura 6). 
Em relação ao $\mathrm{Fe}$, assim como no período chuvoso, é notório que ocorre um acúmulo deste metal ao longo da microbacia do rio Cabeça (P1 a P5) (Figura 7). A bacia tributária que recebe as águas do rio Cabeça apresentou acúmulo de Fe e Al logo após o recebimento das águas de seu afluente. No ponto P7 apresentou concentrações de Fe maiores que no P6 (ponto de coleta anterior ao recebimento das águas do rio Cabeça).

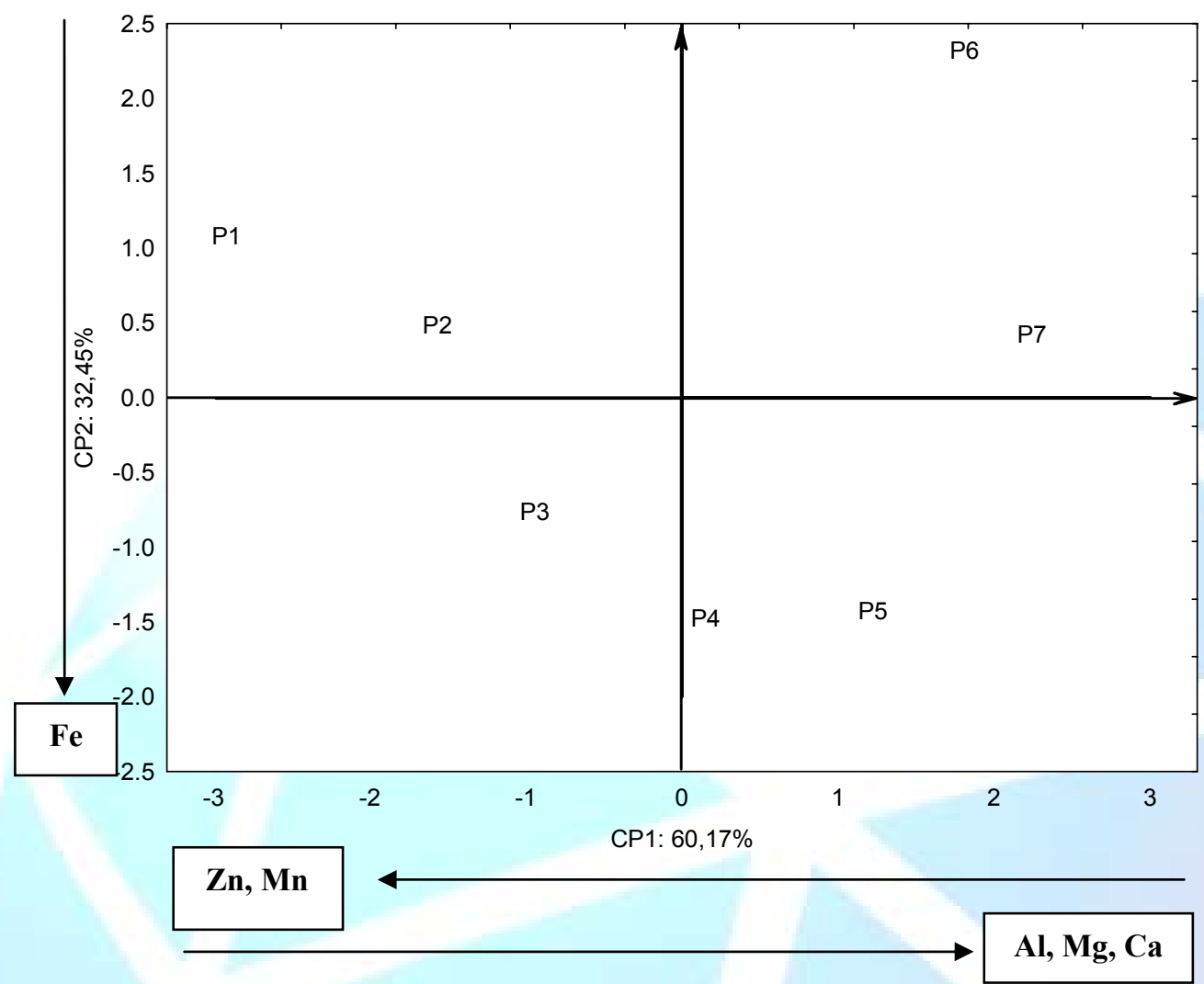

Figura 7. Ordenação pela ACP dos metais nas águas superficiais dos pontos amostrados no período seco.

O cálcio não tem seus valores fixados pela Resolução CONAMA 357/2005 (BRASIL, 2005), mas este elemento foi mensurado devido a sua importância na agricultura e nas cadeias alimentares, assim como o Mg. Verifica-se que o Ca sofreu acréscimo ao longo do rio Cabeça nos dois períodos, seco e chuvoso (Figuras 5 e 6). Os dois pontos no rio Passa Cinco, P6 e P7, seguidos do ponto P5 no rio Cabeça apresentaram os maiores valores de $\mathrm{Ca}$, podendo estar relacionados à localização destes pontos de amostragem em áreas utilizadas para a cultura de cana de açúcar, cuja prática de calagem é frequentemente utilizada. A calagem possui benefícios bastante conhecidos na agricultura e visam principalmente corrigir a acidez; neutralizar os efeitos tóxicos de elementos como o $\mathrm{Al}$ e $\mathrm{Mg}$; aumentar a disponibilidade de alguns nutrientes; contribuir para a melhoria da estrutura do solo e da vida microbiana (BRADY, 1989). Os pontos citados, P5, P6 e P7, também estão localizados em áreas de afloramento de rochas da Formação Irati, unidade em que se extrai o calcário dolomítico utilizado como corretor da acidez de solos (ATLAS AMBIENTAL DA BACIA DO RIO CORUMBATAÍ, 2009). A Figura 3 ilustra as unidades litoestratigráficas da microbacia do rio Cabeça, indicando as áreas predominantes dos afloramentos das rochas e a localização dos pontos de amostragem. 
Quanto ao Mn no período seco, P3 apresentou maiores valores quando comparado aos demais pontos amostrados. Os valores acima do padrão estabelecido pela Resolução CONAMA 357/2005 (BRASIL, 2005) para rios de classe 2, $100 \mu \mathrm{g} . \mathrm{L}^{-1}$, entretanto, foram observados somente no período chuvoso no P1 $\left(165 \mu \mathrm{g} . \mathrm{L}^{-1}\right)$ no mês de janeiro de 2008 ; no P2 $\left(114 \mu \mathrm{g} . \mathrm{L}^{-1}\right)$ e P3 (119 $\left.\mu \mathrm{g} . \mathrm{L}^{-1}\right)$ em dezembro de 2008; no P5 (456 $\left.\mu \mathrm{g} . \mathrm{L}^{-1}\right)$ em fevereiro de 2009 e no P2 $\left(129 \mu \mathrm{g} . \mathrm{L}^{-1}\right)$ e P3 (127 $\left.\mu \mathrm{g} . \mathrm{L}^{-1}\right)$ em março de 2009. O Zn teve seus valores mais elevados no período seco conforme relatado anteriormente. Neste caso, a menor vazão decorrente neste período pode estar influenciando o resultado encontrado. Outra explicação foi dada por Barreto (1999) ao estudar a distribuição de metais no rio Monjolinho na bacia do Alto Jacaré-Guaçu (SP) indicando que o $\mathrm{Zn}$ presente nas águas superficiais era consequência das queimadas das plantações de cana de açúcar, que contribuem para a deposição atmosférica deste metal nos corpos hídrico. As maiores concentrações de $\mathrm{Zn}$, principalmente no período seco, podem estar associadas a esta prática que é mais constante nesta época e devido ao regime de ventos que contribui com a deposição deste metal em áreas adjacentes. Isto pode explicar os maiores valores observados nos pontos P1, P2 e P3. As concentrações de $\mathrm{Zn}$, entretanto, não desrespeitaram os valores estabelecidos pela Resolução CONAMA 357/2005 (BRASIL, 2005) para águas de classe 2.

\section{CONCLUSÕES}

I. Os metais tóxicos $\mathrm{Cr}, \mathrm{Cu}$ e $\mathrm{Ni}$ não foram detectados nos pontos amostrados nas águas do rio Cabeça e Passa Cinco, indicando que não há poluição pelos mesmos.

II. O Cd foi encontrado em $7,1 \%$ e o $\mathrm{Pb}$ em $10,1 \%$ das amostras sendo que os valores estiveram acima do limite máximo estabelecido pela Resolução CONAMA 357/2005 (BRASIL, 2005);

III. O Fe e Al nas águas superficiais na microbacia do rio Cabeça são decorrentes das características pedológicas da área. Já o $\mathrm{Zn}$, apesar dos valores não terem desrespeitado o limite estabelecido pela legislação, pode estar relacionado com a falta de matas ciliares, solo arenoso e intensa ocupação agrícola;

IV. Os valores de $\mathrm{Ca}$ encontrados na foz do rio Cabeça e nos pontos amostrados no rio Passa Cinco provavelmente sofrem influência das práticas agrícolas durante o cultivo de cana-de-açúcar e também das características geológicas da área;

V. Após receber as águas do rio Cabeça nos períodos seco e chuvoso houve acúmulo de Fe e Al no rio Passa Cinco.

VI. Está pesquisa forneceu informações para a melhor compreensão da contribuição das atividades agrícolas na poluição dos corpos hídricos superficiais por metais.

\section{AGRADECIMENTOS}

Os autores agradecem a Coordenação de Aperfeiçoamento de Pessoal de Nível Superior (CAPES) pela concessão de bolsa de doutorado ao primeiro autor.

\section{REFERÊNCIAS}

AL-SHAWI, A. W.; DAHL R. The determination of cadmium and six other heavy metals in nitrate/phosphate fertilizer solution by ion chromatography. Analytica Chimica Acta, Amsterdam, v.391, p.35-42, 1999. 
APHA - AMERICAN PUBLIC HEALTH ASSOCIATION. Standard methods for the examination of water and wastewater. 20 ed. Washington: American Public Health Association, AWWA, WPCF, 1998. 1569p.

ATLAS AMBIENTAL DA BACIA DO RIO CORUMBATAÍ. Rio Claro: Centro de Análise e Planejamento Ambiental - CEAPLA. Instituto de Geociências e Ciências Exatas. UNESP, Campus de Rio Claro. Disponível em: <http://ceapla.rc.unesp.br/atlas/geologia.html >. Acesso em: Nov/2009.

AYDIN, I.; AYDIN, F.; SAYDUT, A.; BAKIRDERE, G.; HAMAMCI, C. Hazardous metal geochemistry of sedimentary phosphate rock used for fertilizer (Mazıdag, SE Anatolia, Turkey). Microchemical Journal, v.96, p.247-251, 2010.

BARRETO, A. S. Estudo da distribuição de metais em ambiente lótico, com ênfase na assimilação pelas comunidades biológicas e na sua quantificação no sedimento e na água. 1999. 276f. Tese (Doutorado em Engenharia Hidráulica e Saneamento) Escola de Engenharia de São Carlos, Universidade de São Paulo, São Carlos, 1999.

BERG, J. M.; TYMOCZKO, J. L.; STRYER, L. Bioquímica. Reimp. Rio de Janeiro: Guanabara Koogan, 2010. 1114p.

BRASIL. Conselho Nacional do Meio Ambiente. Resolução CONAMA n³57. Brasília: MMA, 2005. 23p.

BRADY, N. C. Natureza e propriedades do solo. $7^{\circ}$ ed. Rio de Janeiro: Freitas Bastos, 1989. 898p.

CETESB - Companhia Ambiental do Estado de São Paulo. $1^{\circ}$ ed. São Paulo: CETESB, 1987. 150p.

CORBI, J. J.; STRIXINO, S. T.; SANTOS, A.; DEL GRANDE, M. Diagnóstico ambiental de metais e organoclorados em córregos adjacentes a áreas de cultivo de cana-de-açúcar (Estado de São Paulo, Brasil). Química Nova, v.29, n.1, p.61-65, 2006.

EL-TAHER, A.; ALTHOYAIB, S. S. Natural radio activity levels and heavy metals in chemical and organic fertilizers used in Kingdom of Saudi Arabia. Applied Radiation and Isotopes, v.70 p.290-295, 2012.

GIMENO-GARCÍA, E.; ANDREU, V.; BOLUDA, R. Heavy metals incidence in the application of inorganic fertilizer and pesticides to rice farming soils. Environmental Pollution, Barking, v.92, n.1. p.19-25, 1996.

HARTIGAN, J. A. Clustering algorithms. New York: Wiley, 1975. 351p.

HE, M.; WANG, Z.; TANG, H. Spatial and temporal patterns of acidity and heavy metals in predicting the potential for ecological impact on the Le An river polluted by acid mine drainage.

The Science of the Total Environment, v.206, p.67-77, 1997. 
HE, P. J.; XIAO, Z.; SHAO, L. M.; YU, J. Y.; LEE, D. J. In situ distributions and characteristics of heavy metals in full-scale landfill layers. Journal of Hazardous Materials, v.137, p.1385-1394, 2006.

HOSONO, T.; NAKANO, T.; IGETA, A.; TAYASU, I.; TANAKA, T.; YACHI, S. Impact of fertilizer on a small watershed of Lake Biwa: Use of sulfur and strontium isotopes in environmental diagnosis. Science of the Total Environment, v.384, p.342-354, 2007.

KAUSHIK, A.; KANSAL, A.; MEENA, S.; KUMARI, S.; KAUSHIK, C. P. Heavy metal contamination of river Yamuna, Haryana, India: Assessment by Metal Enrichment Factor of the Sediments. Journal of Hazardous Materials, v.164, p.265-270, 2009.

KIM, J. Y.; LEE, B. T.; SHIN, K. H.; LEE, K. Y.; KIM, K. W.; AN, K. G.; PARK, Y. S.; KIM, J. Y.; KWON, Y. H. Ecological health assessment and remediation of the stream impacted by acid mine drainage of the gwangyang mine area. Environmental Monitoring and Assessment, v.129, p.79-85, 2007.

KRISHNA, A. K.; SATYANARAYANAN, M.; GOVIL, P. K. Assessment of heavy metal pollution in water using multivariate statistical techniques in an industrial area: A case study from Patancheru, Medak District, Andhra Pradesh, India. Journal of Hazardous Materials, v.167, p.366-373, 2009.

LI, S.; XU, Z.; CHENG, X.; ZHANG, Q. Dissolved trace elements and heavy metals in the Danjiangkou Reservoir, China. Environmental Geology, v.55, p.977-983, 2008.

LI, S.; ZHANG, Q. Spatial characterization of dissolved trace elements and heavy metals in the upper Han River (China) using multivariate statistical techniques. Journal of Hazardous Materials, v.176, p.579-588, 2010a.

LI, S.; ZHANG, Q. Risk assessment and seasonal variations of dissolved trace elements and heavy metals in the Upper Han River, China. Journal of Hazardous Materials, v.181, p.1051-1058, $2010 b$.

LU, A.; WANG, J.; QIN, X.; WANG, K.; HAN, P.; ZHANG, S. Multivariate and geostatistical analyses of the spatial distribution and origin of heavy metals in the agricultural soils in Shunyi, Beijing, China. Science of the Total Environment, v.425, p.66-74, 2012.

MACEDA-VEIGA, A.; MONROY, M.; SOSTOA, A. Metal bioaccumulation in the Mediterranean barbell (Barbus meridionalis) in a Mediterranean River receiving effluents from urban and industrial wastewater treatment plants. Ecotoxicology and Environmental Safety, v.76, p.93-101, 2012.

MARTIN, C. W. Recent changes in heavy metal contamination at near-channel positions of the Lahn River, central Germany. Geomorphology, v.139-140, p.452-459, 2012.

MECHE, A.; MARTINS, M. C.; LOFRANO, B. E. S. N.; HARDAWAY, C. J.; MERCHANT. M.; VERDADE, L. Determination of heavy metals by inductively coupled plasma-optical emission spectrometry in fish from the Piracicaba River in Southern Brazil. Microchemical Journal, v.94, p.171-174, 2010. 
MENDES, A. M. S.; DUDA, G. P.; NASCIMENTO, C. W. A.; SILVA, M. O. Bioavailability of cadmium and lead in a soil amended with phosphorus fertilizers. Scientia Agricola, v.63, n.4, p.328-332, 2006.

MORAIS, E.B. Indicadores microbiológicos, metais e índice de qualidade da água (IQA) associados ao uso e ocupação da terra para avaliação da qualidade ambiental da microbacia do rio Cabeça, na bacia do rio Corumbataí, SP. 2010. 138f. Tese (Doutorado em Ciências Biológicas, Área de Concentração em Microbiologia Aplicada), Instituto de Biociências, Universidade Estadual Paulista, Rio Claro, 2010.

MONTEIRO, C. A. F. A dinâmica climática e as chuvas no estado de São Paulo. São Paulo: USP - Instituto de Geografia, 1973. 130p.

NZIGUHEBA, G.; SMOLDE, E. Inputs of trace elements in agricultural soils via phosphate fertilizers in European countries. Science of the Total Environment, v.390, p.53-57, 2008.

PANDEY, P. K.; VERMA, Y.; CHOUBEY, S.; PANDEY, M.; CHANDRASEKHAR, K.

Biosorptive removal of cadmium from contaminated groundwater and industrial effluents.

Bioresource Technology, v.99, p.4420-4427, 2008.

PERTSEMLI, E.; VOUTSA, D. Distribution of heavy metals in Lakes Doirani and Kerkini, Northern Greece. Journal of Hazardous Materials, v.148, p.529-537, 2007.

RAMALHO, J. F. G. P.; SOBRINHO N. M. B. A.; VELLOSO, A. C. X. Acúmulo de metais pesados em solos cultivados com cana-de-açúcar pelo uso contínuo de adubação fosfatada e água de irrigação. Revista Brasileira de Ciências do Solo, v.23, p.971-979, 1999.

RAMALHO, J. F. G. P.; SOBRINHO, N. M. B. A.; VELLOSO, A. C. X. Contaminação da microbacia de Caetés com metais pesados pelo uso de agroquímicos. Pesquisa Agropecuária Brasileira, v.35, n.7, p.1289-1303, 2000.

RAMOS, M. C.; QUINTON, J. N.; TYRREL, S. F. Effects of cattle manure on erosion rates and runoff water pollution by faecal coliforms. Journal of Environmental Management, v.78, p.97$101,2006$.

REZA, R.; SINGH, G. Heavy metal contamination and its indexing approach for river water. International Journal of Environmental Science and Technology, v.7, p.785-792, 2010.

RIBBE, L.; DELGADO, P.; SALGADO, E. FLÜGEL, W. A. Nitrate pollution of surface water induced by agricultural non-point in the Pocochay watershed, Chile. Desalination, v.226, p.13-20, 2008.

RYBICKA, E. H.; ADAMIEC, E.; ALEKSANDER-KWATERCZAK, U. Distribution of trace metals in the Odra River system: Water-suspended matter-sediments. Limnologica, v.35, p.185$198,2005$. 
SABIHA-JAVIED; MEHMOOD, T.; CHAUDHRY, M. M.; TUFAIL, M.; IRFAN, N. Heavy metal pollution from phosphate rock used for the production of fertilizer in Pakistan. Microchemical Journal, v.91, p. 94-99, 2009.

SÃO PAULO. Decreto Estadual no 8.468. São Paulo: Secretaria de Estado do Meio Ambiente. 1976. 75p.

STATSOFT, INC., STATISTICA - Data analysis software system. Version 7.1. www.statsoft.com 2005.

SUTHAR S.; NEMA, A. K.; CHABUKDHARA, M.;. GUPTA S. K. Assessment of metals in water and sediments of Hindon River, India: Impact of industrial and urban discharges. Journal of Hazardous Materials, v.171; p.1088-1095, 2009.

TANG, W.; SHAN, B.; ZHANG, H.; MAO, Z. Heavy metal sources and associated risk in response to agricultural intensification in the estuarine sediments of Chaohu Lake Valley, East China.

Journal of Hazardous Materials, v.176, p.945-951, 2010.

TAUK-TORNISIELO, S. M.; MENEGÁRIO, A. A.; GONÇALVES, F. A. M. Metais nas águas do rio Corumbataí. In: TAUK-TORNISIELO, S. M.; ESQUIERRO, J. C. Bacia do rio Corumbataí aspectos socioeconômicos e ambientais. $1^{\circ}$ ed. Consórcio PCJ. 2008, p.105-118.

TAUK-TORNISIELO, S.M. Pesque-pague. Bacia do Rio Corumbataí. Rio Claro: Centro de Estudos Ambientais. Ed. Divisa. 1ed. 126p. 2010.

VAROL, M.; ŞEN, B. Assessment of nutrient and heavy metal contamination in surface water and sediments of the upper Tigris River, Turkey. Catena, v.92, p.1-10, 2012.

WU, L.; TAN, C.; LIU, L.; ZHU, P.; PENG, C.; LUO, Y.; CHRISTIE, P. Cadmium bioavailability in surface soils receiving long-term applications of inorganic fertilizers and pig manure. Geoderma, v.173-174, p.224-230, 2012.

ZHANG, H.; SHAN, B. Historical records of heavy metal accumulation in sediments and the relationship with agricultural intensification in the Yangtze-Huaihe region, China. Sciebce of the Total Environment, v.399, p-113-120, 2008.

ZHANG, M.; ZHANG, H. Co-transport of dissolved organic matter and heavy metals in soils induced by excessive phosphorus applications. Journal of Environmental Sciences, v.22, n.4, p.598-606, 2010.

ZHOU, Q.; ZHANG, J.; FU, J.; SHI, J.; JIANG, G. Biomonitoring: An appealing tool for assessment of metal pollution in the aquatic ecosystem. Analytica Chimica Acta, v.606, p135-150, 2008. 